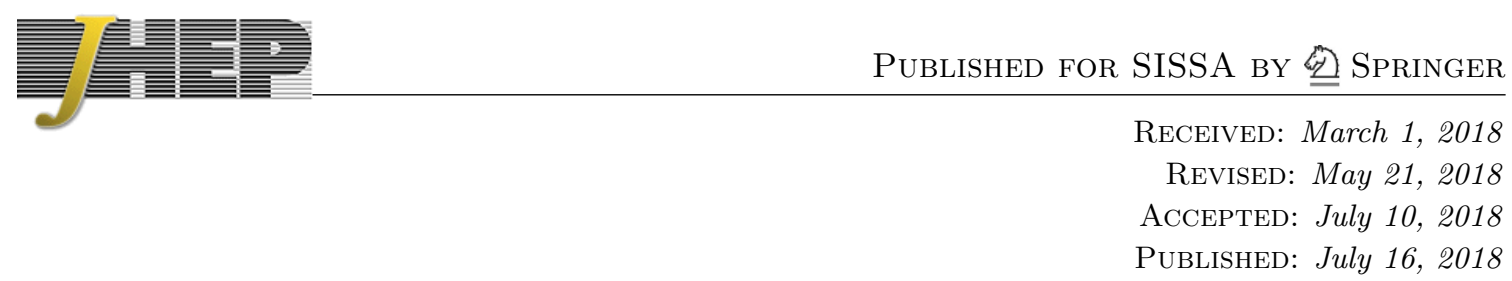

\title{
Mean field quantization of effective string
}

\author{
Yuri Makeenko \\ Institute of Theoretical and Experimental Physics, \\ B. Cheremushkinskaya 25, Moscow, 117218 Russia \\ E-mail: makeenko@itep.ru
}

ABSTRACT: I describe the recently proposed quantization of bosonic string about the meanfield ground state, paying special attention to the differences from the usual quantization about the classical vacuum which turns out to be unstable for $d>2$. In particular, the string susceptibility index $\gamma_{\text {str }}$ is 1 in the usual perturbation theory, but equals $1 / 2$ in the mean-field approximation that applies for $2<d<26$. I show that the total central charge equals zero in the mean-field approximation and argue that fluctuations about the mean field do not spoil conformal invariance.

Keywords: Long strings, Conformal Field Models in String Theory, Bosonic Strings

ArXiv EPrint: 1802.07541 


\section{Contents}

1 Introduction 1

2 The mean-field ground state 3

$\begin{array}{lll}3 & \text { Instability of the classical vacuum } & 7\end{array}$

4 Stability of the mean-field vacuum $\quad 9$

$\begin{array}{lll}5 & \text { The string susceptibility index } & 11\end{array}$

6 Fluctuations about the mean-field $\quad 12$

$\begin{array}{lll}7 & \text { Computation of the central charge } & 15\end{array}$

8 "Semiclassical" correction to the mean field 17

$\begin{array}{lll}8.1 \text { Correction to } T_{z z} & 19\end{array}$

$\begin{array}{ll}8.2 \text { Correction to } S_{\text {eff }} & 20\end{array}$

8.3 Remark on the universality 20

9 Discussion 21

$\begin{array}{ll}\text { A Leading-order explicit computations } & 21\end{array}$

$\begin{array}{lll}\text { A.1 } & T_{a}^{a} & 22\end{array}$

A.2 $T_{z z} \quad 23$

B "Semiclassical" corrections 24

B.1 Contribution to $T_{z z}$

$\begin{array}{ll}\text { B.2 Contribution to } S_{\text {eff }} & 25\end{array}$

C Mathematica programs $\quad \mathbf{2 6}$

C.1 Program for computing diagrams in figure 3 that results in (8.9) 26

C.2 Program for computing diagrams in figure 4 that results in (8.12) 27

\section{Introduction}

Strings or more generally two-dimensional random surfaces have wide applications in physics: from biological membranes to QCD. However, a nonperturbative theory of quantum strings, which goes back to 1980's, makes sense only if the dimension of target space 
$d<2$, where the results both of dynamical triangulation [1-3] and of conformal field theory [4-6] are consistent and agree. For $d>2$ the scaling limit of dynamically triangulated random surfaces is particle-like ${ }^{1}$ rather than string-like because only the lowest mass scales but the string tension does not scale and tends to infinity in the scaling limit. [8] Analogously, the conformal field theory approach does not lead to sensible results for $2<d<26$. [4-6] The conclusion was that quantum string does not exist nonperturbatively for $2<d<26$, while it beautifully works for $d<2$. However, we understand that strings do exist as physical objects in $d=4$ space-time dimensions.

A potential way out was to adopt the viewpoint that string is not a fundamental object but is rather formed by more fundamental degrees of freedom. This philosophy perfectly applies to QCD string, ${ }^{2}$ where these are fluxes of the gauge field. String description makes sense only for the distances larger than the confinement scale. For shorter distances the quark-gluon degrees of freedom are more relevant due to asymptotic freedom. This picture is well justified both by experiment and by lattice simulations. The string tachyon which is a short-distance phenomenon does not show up in the QCD spectrum.

A breakthrough along this line is due to the "effective string" philosophy [10], which works perturbatively order by order in the inverse string length (for recent advances see [1115]). Then string quantization is consistent even below the critical dimension $(d=26$ for the relativistic bosonic string) and a few leading orders reproduce [10, 16, 17] the AlvarezArvis ground-state energy [18-20]. In this Paper I shall pay much attention to this issue.

In the recent series of papers [21-24] it has been understood why lattice string formulations resulted in the particle-like continuum limit. A nonperturbative mean-field solution of the Nambu-Goto string showed that the usual classical vacuum about which string is quantized is unstable for $d>2$, while another nonperturbative vacuum is stable for $2<d<26$, like it happens in the well-known example of the two-dimensional $O(N)$ sigma-model. For the true ground state the value of the metric at the string worldsheet $\left(\rho_{a b}=\bar{\rho} \delta_{a b}\right.$ in the conformal gauge) becames infinite in the scaling limit. For this reason an infinite amount of stringy modes (which is $\propto \bar{\rho} / a^{2}$ with $a$ being a UV cutoff) can be reached even at the distances of order $a$. That was in contrast to the usual continuum limit in quantum field theory, where the amount of degrees of freedom can be infinite only if the correlation length is infinite. The discovered phenomenon is specific to theories with diffeomorphism invariance and was called the Lilliputian continuum limit.

The task of this Paper is to analyze properties of the mean-field vacuum which plays the role of a "classical" string ground state and "quantum" fluctuations about it. I put here quotes to emphasize this state is a genuine nonperturbative quantum state from the viewpoint of the usual semiclassical expansion in $\alpha^{\prime}$ about the classical vacuum. We thus perform a resummation of this expansion with the leading order given by the sum of bubblelike diagrams. An analogy with the two-dimensional $O(N)$ sigma-model at large $N$ can be again instructive. I shall pay special attention to a comparison with the KnizhnikPolyakov-Zamolodchikov (KPZ)-David-Distler-Kawai (DDK) results [4-6] for the parturbative vacuum, which is applicable for $d<2$.

\footnotetext{
${ }^{1} \mathrm{~A}$ detailed description can be found in the book [7].

${ }^{2}$ For a brief introduction see e.g. [9].
} 
It will be shown in the Paper that the total central charge of the system vanishes in the mean-field approximation which is thus consistent in noncritical dimension $2<d<26$. This is in contrast to the old canonical quantization which works only in the critical dimension $d=26$ and compliments the effective-string approach of Polchinski-Strominger, where the consistency is explicitly demonstrated to a few lower orders of the perturbative expansion $[10,16,17]$. I then analyze a "semiclassical" correction to the mean-field approximation and show that it does not spoil conformal invariance in spite of logarithmic infrared divergences caused by the propagator of a massless field, which cancel in the sum of diagrams.

This Paper is organized as follows. In sections 2, 3, 4 I review the results [21-23] which form a background for further investigations. Section 5 is devoted to the computation of the mean-field value of the string susceptibility index $\gamma_{\mathrm{str}}=1 / 2$ and its comparison to the perturbative value $\gamma_{\text {str }}=1$. In section 6 I formulate a general procedure for expanding about the mean field and describe Pauli-Villars' regulation for computing the energy-momentum tensor and its trace anomaly, which does not rely on approximating the involved determinants by (the exponential of) the conformal anomaly. It section 7 I compute the total central charge of the system in the mean-field approximation and show that it vanishes for $2<d<26$. Section 8 is devoted to the "semiclassical" expansion about the mean field. I show that logarithmic infrared divergences which might spoil conformal invariance are mutually canceled. The results obtained and tasks for the future are discussed in section 9 . Some explicit computations are presented in appendices A, B by using the Mathematica programs from appendix C.

\section{The mean-field ground state}

We consider a closed bosonic string in target space with one compactified dimension of circumference $\beta$. The string wraps once around this compact dimension and propagates through the distance $L$. The string world-sheet has thus topology of a cylinder. There is no tachyon for such a string configuration, if $\beta$ is larger than a certain value to guarantee that the classical energy of the string dominates over the energy of zero-point fluctuations.

The Nambu-Goto string action is given by the area of the surface embedded in target space. It is highly nonlinear in the embedding-space coordinate $X^{\mu}$. To make it quadratic in $X^{\mu}$, we rewrite it, introducing a Lagrange multiplier $\lambda^{a b}$ and an independent intrinsic metric $\rho_{a b}$, as $^{3}$

$$
S=K_{0} \int \mathrm{d}^{2} \omega \sqrt{\operatorname{det} \partial_{a} X \cdot \partial_{b} X}=K_{0} \int \mathrm{d}^{2} \omega \sqrt{\operatorname{det} \rho}+\frac{K_{0}}{2} \int \mathrm{d}^{2} \omega \lambda^{a b}\left(\partial_{a} X \cdot \partial_{b} X-\rho_{a b}\right),
$$

where $K_{0}$ stands for the bare string tension. The equivalence of the two formulations can be proven by path integrating over the functions $\lambda^{a b}(\omega)$ and $\rho_{a b}(\omega)$ which take on imaginary and real values, respectively.

It is convenient to choose the world-sheet coordinates $\omega_{1}$ and $\omega_{2}$ inside an $\omega_{L} \times \omega_{\beta}$ rectangle in the parameter space. Then the classical solution $X_{\mathrm{cl}}^{\mu}$ minimizing the action (2.1)

\footnotetext{
${ }^{3}$ We denote $\operatorname{det} \rho=\operatorname{det} \rho_{a b}$ and $\operatorname{det} \lambda=\operatorname{det} \lambda^{a b}$.
} 
linearly depends on $\omega$

$$
X_{\mathrm{cl}}^{1}=\frac{L}{\omega_{L}} \omega_{1}, \quad X_{\mathrm{cl}}^{2}=\frac{\beta}{\omega_{\beta}} \omega_{2}
$$

The classical value of $\rho_{a b}$ coincides with the classical induced metrics

$$
\left[\rho_{\mathrm{cl}}\right]_{a b}=\partial_{a} X_{\mathrm{cl}} \cdot \partial_{b} X_{\mathrm{cl}}=\operatorname{diag}\left(\frac{L^{2}}{\omega_{L}^{2}}, \frac{\beta^{2}}{\omega_{\beta}^{2}}\right)
$$

which becomes diagonal for

$$
\omega_{\beta}=\frac{\beta}{L} \omega_{L}
$$

The classical value of $\lambda^{a b}$ reads

$$
\lambda_{\mathrm{cl}}^{a b}=\rho_{\mathrm{cl}}^{a b} \sqrt{\operatorname{det} \rho_{\mathrm{cl}}}
$$

and simplifies to $\lambda_{\mathrm{cl}}^{a b}=\delta^{a b}$ if eq. (2.4) is satisfied.

We apply the path-integral quantization to account for quantum fluctuations of the $X$-fields by splitting $X^{\mu}=X_{\mathrm{cl}}^{\mu}+X_{\mathrm{q}}^{\mu}$ and then performing the Gaussian path integral over $X_{\mathrm{q}}^{\mu}$. We thus obtain the action, governing the fields $\lambda^{a b}$ and $\rho_{a b}$,

$$
\begin{aligned}
S_{\text {ind }} & =K_{0} \int \mathrm{d}^{2} \omega \sqrt{\operatorname{det} \rho}+\frac{K_{0}}{2} \int \mathrm{d}^{2} \omega \lambda^{a b}\left(\partial_{a} X_{\mathrm{cl}} \cdot \partial_{b} X_{\mathrm{cl}}-\rho_{a b}\right)+\frac{d}{2} \operatorname{tr} \log (-\mathcal{O}), \\
\mathcal{O} & =\frac{1}{\sqrt{\operatorname{det} \rho}} \partial_{a} \lambda^{a b} \partial_{b} .
\end{aligned}
$$

The operator $\mathcal{O}$ reproduces the usual two-dimensional Laplacian $\Delta$ for $\lambda^{a b}$ given by the right-hand side of eq. (2.5). Its determinant is to be computed with the Dirichlet boundary condition imposed. Quantum observables are determined by the path integral over $\lambda^{a b}$ and $\rho_{a b}$ with the action (2.8), which runs as is already mentioned over imaginary $\lambda^{a b}(\omega)$ and real $\rho_{a b}(\omega)$. The action (2.6) is often called the induced (or emergent) action to be distinguished from the effective action which is usually associated with slowly varying fields in the low-momentum limit.

It is convenient to fix the conformal gauge when $\rho_{a b}=\rho \delta_{a b}$, so that $\sqrt{\operatorname{det} \rho}=\rho$. Then the log of the determinant of the ghost operator [25]

$$
\left[\mathcal{O}_{\mathrm{gh}}\right]_{b}^{a}=\Delta_{b}^{a}-\frac{1}{2}\left(\Delta_{b}^{a} \log \rho\right)
$$

is to be added to the induced action (2.6) [or (4.11) below]. The operator (2.7) acts on twodimensional vector functions whose one component obeys the Dirichlet boundary condition and the other obeys the Robin boundary condition [26, 27]. The subtleties associated with the boundary conditions will be inessential both for the matter and ghost determinants for $L \gg \beta$ when only the bulk terms survive.

We shall describe in section 6 how to accurately compute the determinants using the Pauli-Villars regularization but let us assume for a moment that $\lambda^{a b}(\omega)=\bar{\lambda} \delta^{a b}$ and $\rho_{a b}(\omega)=\bar{\rho} \delta_{a b}$ with constant $\bar{\lambda}$ and $\bar{\rho}$. As we shall see these constant values of $\bar{\lambda}$ and $\bar{\rho}$ are what is needed for the mean-field approximation. 
The computation of the matter and ghost determinants for constant $\bar{\lambda}$ and $\bar{\rho}$ is presently an exercise in string theory courses with the result

$$
S_{\mathrm{eff}}=\frac{K_{0}}{2} \bar{\lambda}\left(\frac{L^{2}}{\omega_{L}^{2}}+\frac{\beta^{2}}{\omega_{\beta}^{2}}\right) \omega_{L} \omega_{\beta}+K_{0}(1-\bar{\lambda}) \bar{\rho} \omega_{L} \omega_{\beta}-\left(\frac{d}{2 \bar{\lambda}}-1\right) \Lambda^{2} \bar{\rho} \omega_{L} \omega_{\beta}-\frac{\pi(d-2)}{6} \frac{\omega_{L}}{\omega_{\beta}}
$$

for $L \gg \beta$. Here $\Lambda^{2}$ cuts off eigenvalues of the operators involved. The first and second terms on the right-hand side are classical contributions, while the sign of the third term is negative for $d / \bar{\lambda}>2$ to comply with positive entropy. Technically, it comes as the product of the eigenvalues divided by $\Lambda$, where every multiplier is less than 1 . The last term is known as the Lüscher term which is due to the Casimir energy of zero-point fluctuations. Its negative sign is intimately linked to the presence of the tachyon.

The next step is to minimize (2.8) over $\bar{\lambda}, \bar{\rho}$ to find the mean-field configuration which describes the string ground state. The difference from the classical ground state (2.2), (2.3), (2.5) is that we now minimize the action, taking into account the determinants coming from $X^{\mu}$ and ghosts, while the classical (perturbative) ground state minimizes the classical action. Additionally, similarly to the classical case we have to minimize (2.8) over the ratio $\omega_{\beta} / \omega_{L}$ which plays the role of the modular parameter of the cylinder. This guarantees that $\rho_{a b}$ and $\lambda^{a b}$ are diagonal as is required by the conformal gauge. We shall return to this issue soon.

The minimum of (2.8) is remarkable simple [21-23]

$$
\begin{aligned}
\bar{\lambda} & =\frac{1}{2}+\frac{\Lambda^{2}}{2 K_{0}}+\sqrt{\frac{1}{4}\left(1+\frac{\Lambda^{2}}{K_{0}}\right)^{2}-\frac{d \Lambda^{2}}{2 K_{0}}}, \\
\bar{\rho} & =\frac{\left(\beta^{2}-\frac{\pi(d-2)}{6 K_{0} \bar{\lambda}}\right)}{\omega_{\beta}^{2}} \frac{\bar{\lambda}}{\sqrt{\left(1+\frac{\Lambda^{2}}{K_{0}}\right)^{2}-\frac{2 d \Lambda^{2}}{K_{0}}}} \\
\omega_{\beta} & =\frac{\omega_{L}}{L} \sqrt{\beta^{2}-\frac{\pi(d-2)}{3 K_{0} \bar{\lambda}}}
\end{aligned}
$$

The value of the action (2.8) at the minimum (2.9) is

$$
S_{\mathrm{mf}}=K_{0} \bar{\lambda} L \sqrt{\beta^{2}-\frac{\pi(d-2)}{3 K_{0} \bar{\lambda}}} .
$$

The meaning of the above minimization procedure is clear: we have constructed a saddle-point approximation to the path integral, which takes into account an infinite set of diagrams of perturbation theory about the classical vacuum. This approach is quite similar to that ${ }^{4}$ in the two-dimensional $O(N)$ sigma-model, where one sums up bubble diagrams of the $1 / N$-expansion by introducing the Lagrange multiplier $u$ to resolve the constraint $\vec{n}^{2}=1$. After integration over the fields $\vec{n}$ one obtains an induced action as a functional

\footnotetext{
${ }^{4}$ See e.g. the book [28].
} 
of $u$, whose minimum determines the exact vacuum state as $N \rightarrow \infty$. For finite $N$ the fluctuations of $u$ about this mean-field vacuum have to be included, but they are small even at $N=3$ because, roughly speaking, there is only one $u$ while the induced action is of order $N$, i.e. large as is needed for a saddle point. Alternatively, the perturbative vacuum $\vec{n}_{\mathrm{cl}}=(1,0, \ldots, 0)$ possesses an $O(N-1)$ symmetry rather than the $O(N)$ symmetry as the saddle-point vacuum does and the fields $\vec{n}$ fluctuate strongly. For our case the number of fields $X^{\mu}$ in the sigma model (2.1) is $d$, so the saddle-point is justified by $K_{0} \sim d \rightarrow \infty$. At finite $d$ the saddle-point solution (2.9) is associated with the mean-field approximation.

The minimization of the action (2.8) over $\omega_{\beta} / \omega_{L}$ can be now understood as follows. In the mean-field approximation we consider the action to be large, doing all integrals by the saddle point, including the integral over the modular parameter, which is present for the cylinder topology.

A few comments concerning the solution (2.9) are in order:

- Equation (2.9a) is well-defined if the bare string tension $K_{0}>K_{*}$ given by

$$
K_{*}=\left(d-1+\sqrt{d^{2}-2 d}\right) \Lambda^{2} .
$$

At this critical value of $K_{0}$ the square root in (2.9a) vanishes.

- The classical vacuum (2.2), (2.3), (2.5) is recovered by (2.9) as $K_{0} \rightarrow \infty$, while the expansion in $1 / K_{0}$ makes sense of the semiclassical (perturbative) expansion about this vacuum. The usual one-loop results are recovered to order $1 / K_{0}$.

- The large- $d$ ground-state energy $[18],{ }^{5}$ where an analytic regularization was used, are recovered by eq. (2.10) for $\Lambda^{2}=0$. Analogously, the ground-state energy obtained by the old canonical quantization [19] is reproduced by our mean-field approximation. This is not surprising because fluctuations of $\rho$ are ignored in the canonical quantization.

- Equation (2.10) is well-defined for $\beta$ larger than $\sqrt{\pi(d-2) / 3 K_{0} \bar{\lambda}} \sim 1 / \Lambda$, but becomes imaginary otherwise. The singularity was linked [18-20] to the tachyon mass squared.

- The metric (2.9b) becomes infinite when $K_{0} \rightarrow K_{*}$ given by eq. (2.11). This is crucial for constructing the scaling limit.

At the classical level $\rho_{\mathrm{cl}}$ coincides with the induced metric as is displayed in (2.3). In the mean-field approximation it is superseded by

$$
\bar{\rho}_{a b}=\left\langle\partial_{a} X \cdot \partial_{b} X\right\rangle,
$$

where the average is understood in the sense of the path integral over $X^{\mu}$. Equation (2.12) follows from the minimization of the effective action over $\lambda^{a b}$. Thus, in the mean-field approximation $\bar{\rho}$ coincides with the averaged induced metric.

\footnotetext{
${ }^{5}$ The original computation [18] used the Nambu-Goto string. How the same result can be obtained for the Polyakov string is shown in [29].
} 


\section{Instability of the classical vacuum}

The usual semiclassical (or one-loop) correction to the classical ground-state energy due to zero-point fluctuations [30] is described in textbooks. The sum of the two reads

$$
S_{1 l}=\left[K_{0}-\frac{(d-2)}{2} \Lambda^{2}\right] L \beta-\frac{\pi(d-2)}{6} \frac{L}{\beta} .
$$

To make the bulk part of (3.1) finite, it is usually introduced the renormalized string tension

$$
K_{R}=K_{0}-\frac{(d-2)}{2} \Lambda^{2}
$$

which is kept finite as $\Lambda \rightarrow \infty$. Then it is assumed that it works order by order of the perturbative expansion about the classical vacuum, so that $K_{R}$ can be made finite by fine tuning $K_{0}$.

We see however from eq. (2.10) how it may not be case. The right-hand side of eq. (2.10) never vanishes with changing $K_{0}$. The point of view on eq. (3.1) should be that for $d>2$ the one-loop correction simply lowers the energy of the classical ground state which therefore may be unstable.

As we show in the next section, the action (2.8) indeed increases if we add a constant imaginary addition $\delta \lambda$ to $\bar{\lambda}$. However, the sum of the two linear in $\bar{\rho}$ terms in eq. (2.8) vanishes for $\bar{\lambda}$ given by eq. (2.9a), so the action does not depend on $\bar{\rho}$ at the minimum. This reminds a valley in the problem of spontaneous symmetry breaking.

To investigate it, we proceed in the standard way, adding to the action the source term

$$
S_{\text {src }}=\frac{K_{0}}{2} \int \mathrm{d}^{2} \omega j^{a b} \rho_{a b}
$$

and defining the field

$$
\rho_{a b}(j)=-\frac{2}{K_{0}} \frac{\delta}{\delta j^{a b}} \log Z
$$

Minimizing the action with the source term added for constant $j^{a b}=j \delta^{a b}$, we find [23]

$$
\bar{\lambda}(j)=\frac{1}{2}\left(1+j+\frac{\Lambda^{2}}{K_{0}}\right)+\sqrt{\frac{1}{4}\left(1+j+\frac{\Lambda^{2}}{K_{0}}\right)^{2}-\frac{d \Lambda^{2}}{2 K_{0}}}
$$

and

$$
\bar{\rho}(j)=\frac{1}{2}+\frac{1+j+\frac{\Lambda^{2}}{K_{0}}}{\sqrt{\left(1+j+\frac{\Lambda^{2}}{K_{0}}\right)^{2}-\frac{2 d \Lambda^{2}}{K_{0}}}}
$$

in the mean-field approximation for $\omega_{L}=L$ and $\omega_{\beta}=\beta \gg 1 \sqrt{K_{0}}$. Inverting eq. (3.6), we obtain

$$
j(\bar{\rho})=-1-\frac{\Lambda^{2}}{K_{0}}+\sqrt{\frac{d \Lambda^{2}}{2 K_{0}}} \frac{(2 \bar{\rho}-1)}{\sqrt{\bar{\rho}(\bar{\rho}-1)}} .
$$


To understand the properties of the vacuum, we compute an "effective potential" by performing the Legendre transformation

$$
\Gamma(\bar{\rho})=-\frac{1}{K_{0} L \beta} \log Z-j(\bar{\rho}) \bar{\rho},
$$

like in the studies of symmetry breaking in quantum field theory.

In the mean-field approximation we then obtain

$$
\Gamma(\bar{\rho})=\left(1+\frac{\Lambda^{2}}{K_{0}}\right) \bar{\rho}-\sqrt{\frac{2 d \Lambda^{2}}{K_{0}} \bar{\rho}(\bar{\rho}-1)} .
$$

Note that

$$
-\frac{\partial \Gamma(\bar{\rho})}{\partial \bar{\rho}}=j(\bar{\rho})
$$

with $j(\bar{\rho})$ given by eq. (3.7) as it should.

Near the classical vacuum we have $0<\bar{\rho}-1 \ll 1$ and the potential (3.9) decreases with increasing $\bar{\rho}$ because the second term on the right-hand side has the negative sign, demonstrating an instability of the classical vacuum. If $K_{0}>K_{*}$ given by eq. (2.11), the potential (3.9) linearly increases with $\bar{\rho}$ for large $\bar{\rho}$ and thus has a (stable) minimum at

$$
\bar{\rho}(0)=\frac{1}{2}+\frac{1+\frac{\Lambda^{2}}{K_{0}}}{2 \sqrt{\left(1+\frac{\Lambda^{2}}{K_{0}}\right)^{2}-\frac{2 d \Lambda^{2}}{K_{0}}}}
$$

which is the same as $(2.9 \mathrm{~b})$ for $\omega_{\beta}=\beta \gg 1 / \sqrt{K_{0}}$. Near the minimum we have

$$
\begin{aligned}
\Gamma(\bar{\rho}) & =\left[\left(1+\frac{\Lambda^{2}}{K_{0}}\right)^{2}-\frac{2 d \Lambda^{2}}{K_{0}}\right]^{1 / 2}+\frac{K_{0}}{2 d \Lambda^{2}}\left[\left(1+\frac{\Lambda^{2}}{K_{0}}\right)^{2}-\frac{2 d \Lambda^{2}}{K_{0}}\right]^{3 / 2}(\Delta \bar{\rho})^{2}+\mathcal{O}\left((\Delta \bar{\rho})^{3}\right), \\
\Delta \bar{\rho} & =\bar{\rho}-\bar{\rho}(0) .
\end{aligned}
$$

The coefficient in front of the quadratic term is positive for $K_{0}>K_{*}$ which explicitly demonstrates the (global) stability of the mean-field minimum (2.9b).

The situation is different for $d<2$, where quantum corrections increase the vacuum energy. For this reason the classical vacuum is energetically favorable to the mean-field one. It is explicitly seen for $d<0$ from eq. (3.9) where $\bar{\rho}-1$ has to be negative. The function $\Gamma(\bar{\rho})$ then increases with decreasing $\bar{\rho}$ near $\bar{\rho}=1$ and the mean-field solution is a maximum, not a minimum.

The conclusion of this section is that the classical vacuum is not stable for $d>2$ where the mean-field vacuum is energetically favorable. This reminds spontaneous generation of $\bar{\rho}$ in quantum field theory. The situation is opposite for $d<2$, where the classical vacuum has lower energy than the mean-field vacuum. 


\section{Stability of the mean-field vacuum}

Let us now consider stability of the mean-field vacuum under wavy fluctuations, when

$$
\rho(\omega)=\bar{\rho}+\delta \rho(\omega), \quad \lambda^{a b}(\omega)=\bar{\lambda} \delta^{a b}+\delta \lambda^{a b}(\omega)
$$

with $\omega$-dependent $\delta \rho$ and $\delta \lambda$.

The divergent part of the effective action reads [23]

$$
\begin{aligned}
S_{\text {div }} & =\int \mathrm{d}^{2} \omega\left[\frac{K_{0}}{2} \lambda^{a b} \partial_{a} X_{\mathrm{cl}} \cdot \partial_{b} X_{\mathrm{cl}}+K_{0} \rho\left(1-\frac{1}{2} \lambda^{a a}\right)-\frac{d \Lambda^{2} \rho}{2 \sqrt{\operatorname{det} \lambda}}+\Lambda^{2} \rho\right], \\
\lambda^{a a} & =\lambda^{11}+\lambda^{22} .
\end{aligned}
$$

For constant $\lambda^{a b}=\bar{\lambda} \delta^{a b}$ and $\rho=\bar{\rho}$ this reproduces the divergent part of eq. (2.8) above.

Expanding to quadratic order in fluctuations

$$
\begin{aligned}
\sqrt{\operatorname{det}\left(\bar{\lambda} \delta^{a b}+\delta \lambda^{a b}\right)} & =\bar{\lambda}+\frac{1}{2} \delta \lambda^{a a}-\delta \lambda_{2}+\mathcal{O}\left((\delta \lambda)^{3}\right) \\
\delta \lambda_{2} & =\frac{1}{8 \bar{\lambda}}\left(\delta \lambda_{11}-\delta \lambda_{22}\right)^{2}+\frac{1}{2 \bar{\lambda}}\left(\delta \lambda_{12}\right)^{2}
\end{aligned}
$$

we find from (4.2)

$$
S_{\text {div }}^{(2)}=-\frac{d \Lambda^{2} \bar{\rho}}{2 \bar{\lambda}} \int \mathrm{d}^{2} \omega \delta \lambda_{2}-\left(K_{0}-\frac{d \Lambda^{2}}{2 \bar{\lambda}^{2}}\right) \int \mathrm{d}^{2} \omega \delta \rho \frac{\delta \lambda^{a a}}{2}-\frac{d \Lambda^{2} \bar{\rho}}{2 \bar{\lambda}^{3}} \int \mathrm{d}^{2} \omega\left(\frac{\delta \lambda^{a a}}{2}\right)^{2} .
$$

The first term on the right-hand side of eq. (4.4) plays a very important role for dynamics of quadratic fluctuations. Because the path integral over $\lambda^{a b}$ goes parallel to imaginary axis, i.e. $\delta \lambda^{a b}$ is pure imaginary, the first term is always positive. Moreover, its exponential plays the role of a (functional) delta-function as $\Lambda \rightarrow \infty$, forcing $\delta \lambda^{a b}=\delta \lambda \delta^{a b}$. The same is true for a constant part of $\delta \lambda^{a b}$.

For the effective action to the second order in fluctuations we then find the following quadratic form:

$$
\delta S_{2}=\int \frac{\mathrm{d}^{2} p}{(2 \pi)^{2}}\left[A_{\rho \rho} \frac{\delta \rho(p) \delta \rho(-p)}{\bar{\rho}^{2}}+2 A_{\rho \lambda} \frac{\delta \rho(p) \delta \lambda(-p)}{\bar{\rho} \bar{\lambda}}+A_{\lambda \lambda} \frac{\delta \lambda(p) \delta \lambda(-p)}{\bar{\lambda}^{2}}\right]
$$

where

$$
\begin{aligned}
& A_{\rho \rho}=\frac{(26-d) p^{2}}{96 \pi} \\
& A_{\rho \lambda}=-\frac{1}{2}\left(K_{0}-\frac{d \Lambda^{2}}{2 \bar{\lambda}^{2}}\right) \bar{\rho} \bar{\lambda}-\frac{d p^{2}}{48 \pi} \\
& A_{\lambda \lambda}=-\frac{d \Lambda^{2} \bar{\rho}}{2 \bar{\lambda}}-\frac{d p^{2}}{32 \pi} \log \frac{c p^{2}}{\Lambda^{2} \bar{\rho}} .
\end{aligned}
$$

Here $c$ is a regularization-dependent constant. 
In the scaling limit, where $[21-23]$

$$
K_{0} \rightarrow K_{*}+\frac{K_{R}^{2}}{2 \Lambda^{2} \sqrt{d^{2}-2 d}}
$$

as $\Lambda \rightarrow \infty$ keeping the renormalized string tension $K_{R}$ fixed, we have

$$
K_{0}-\frac{d \Lambda^{2}}{2 \bar{\lambda}^{2}} \rightarrow K_{R}\left(1+\sqrt{1-\frac{2}{d}}\right),
$$

so only $A_{\lambda \lambda}$ diverges as $\Lambda^{2}$. Therefore, typical $\delta \lambda \sim 1 / \Lambda$ so that $\lambda^{a b}$ is localized at the value

$$
\bar{\lambda}^{a b}=\bar{\lambda} \delta^{a b} .
$$

This is quite similar to what is shown in the book [31] for the fluctuations about the classical vacuum. Thus only $\rho$ fluctuates.

Equation (4.9) holds in the conformal gauge, where $\rho^{a b} \sqrt{\operatorname{det} \rho}=\delta^{a b}$. In the general case the field $\lambda^{a b}(\omega)$ is localized at the value

$$
\bar{\lambda}^{a b}=\bar{\lambda} \rho^{a b} \sqrt{\operatorname{det} \rho}
$$

where $\bar{\lambda}$ is constant for the world-sheet parametrization in use.

We can therefore rewrite the right-hand side of eq. (2.1) in the scaling limit as

$$
S=K_{0}(1-\bar{\lambda}) \int \mathrm{d}^{2} \omega \sqrt{\operatorname{det} \rho}+\frac{K_{0} \bar{\lambda}}{2} \int \mathrm{d}^{2} \omega \sqrt{\operatorname{det} \rho} \rho^{a b} \partial_{a} X \cdot \partial_{b} X
$$

which reproduces the Polyakov string formulation [25] for $\bar{\lambda}=1$. As shown in $[21,22]$ the action (4.11) is consistent only for a certain value of $\bar{\lambda}$ which is regularization-dependent. One has $\bar{\lambda}=1$ for the zeta-function regularization but $\bar{\lambda}<1$ for the proper-time regularization or the Pauli-Villars regularization.

A subtlety with the computation of the determinants in the conformal gauge is that $X^{\mu}$ and $\rho$ do not interact in the action (4.11) since

$$
S=K_{0}(1-\bar{\lambda}) \int \mathrm{d}^{2} \omega \sqrt{\hat{g}} \rho+\frac{K_{0} \bar{\lambda}}{2} \int \mathrm{d}^{2} \omega \sqrt{\hat{g}} \hat{g}^{a b} \partial_{a} X \cdot \partial_{b} X
$$

in the conformal gauge $\rho_{a b}=\hat{g}_{a b} \rho$. Here $\hat{g}_{a b}$ is a fiducial metric which we can set $\hat{g}_{a b}=\delta_{a b}$ without loss of generality.

But the dependence of the determinants on $\rho$ appears because the world-sheet regularization

$$
\varepsilon=\frac{1}{\Lambda^{2} \sqrt{\operatorname{det} \rho}}=\frac{1}{\Lambda^{2} \rho}
$$

depends on $\rho$ owing to diffeomorphism invariance. For smooth $\rho$ the determinants are given by the usual conformal anomaly [25]. An advantage of using the Pauli-Villars regularization in the conformal gauge is that the implicit dependence on the metric becomes explicit as is described in section 6 . 
Integrating over the matter and ghost fields, we arrive for $\hat{g}_{a b}=\delta_{a b}$ to the induced action

$$
\begin{aligned}
S_{\mathrm{ind}}= & \frac{K_{0} \bar{\lambda}}{2} \int \mathrm{d}^{2} \omega \delta^{a b} \partial_{a} X_{\mathrm{cl}} \cdot \partial_{b} X_{\mathrm{cl}}+K_{0}(1-\bar{\lambda}) \int \mathrm{d}^{2} \omega \rho \\
& +\left.\frac{d}{2} \operatorname{tr} \log \left(-\frac{\bar{\lambda}}{\rho} \partial^{2}\right)\right|_{\mathrm{reg}}-\left.\frac{1}{2} \operatorname{tr} \log \left(\left[-\mathcal{O}_{\mathrm{gh}}\right]_{b}^{a}\right)\right|_{\mathrm{reg}},
\end{aligned}
$$

where the ghost operator is displayed in eq. (2.7). Evaluating the determinants, we find for smooth $\rho$

$$
\begin{aligned}
S_{\text {eff }}= & \frac{K_{0} \bar{\lambda}}{2}\left(\frac{L^{2} \omega_{\beta}}{\omega_{L}}+\frac{\beta^{2} \omega_{L}}{\omega_{\beta}}\right)+\left[K_{0}(1-\bar{\lambda})-\left(\frac{d}{2 \bar{\lambda}}-1\right) \Lambda^{2}\right] \int \mathrm{d}^{2} \omega \rho \\
& +\frac{26-d}{96 \pi} \int \mathrm{d}^{2} \omega\left(\partial_{a} \log \rho\right)^{2}
\end{aligned}
$$

which for $\bar{\lambda}=1$ reproduces the usual result.

We see from eq. (4.15) (as well as from eq. (4.5) with $\delta \lambda=0$ ) that the action, describing fluctuations of the metric, is positive only for $d<26$ and becomes negative if $d>26$. Thus, as far as the local stability of the action under wavy fluctuations is concern, it is the same about the mean-field vacuum as about the usual classical vacuum. This instability is probably linked to the presence of negative-norm states for $d>26[32,33]$.

\section{The string susceptibility index}

A very important characteristics of the string dynamics is the string susceptibility index $\gamma_{\text {str }}$ which characterizes the string entropy and is determined from the preexponential in the number of surfaces of fixed area $A$ by

$$
\mathrm{e}^{-F(A)} \equiv\left\langle\delta\left(\int \mathrm{d}^{2} z \rho-A\right)\right\rangle \stackrel{A \rightarrow \infty}{\propto} A^{\gamma_{\mathrm{str}}-2} \mathrm{e}^{C A},
$$

where $C$ is a nonuniversal constant. $F(A)$ on the left-hand side has the meaning of the Helmholtz free energy of a canonical ensemble at fixed area $A$. Introducing the Lagrange multiplier, we rewrite (5.1) as

$$
\left\langle\delta\left(\int \mathrm{d}^{2} z \rho-A\right)\right\rangle=\left\langle\int \mathrm{d} j \mathrm{e}^{j\left(\int \mathrm{d}^{2} z \rho-A\right)}\right\rangle,
$$

where the integral over $j$ runs parallel to the imaginary axis. This $j$ is the same as introduced in section 3 except for the integral over $j$.

Let us first consider the integrand. The saddle-point solution is given by eq. (3.6). Then the integrand in (5.2) has an extremum at $j(A)$ given by eq. (3.7) with $\bar{\rho}$ substituted by $A / A_{\min }, A_{\min }=L \beta$. Expanding about the extremum, we find [24]

$$
\begin{aligned}
\frac{j A}{K_{0} A_{\min }}-\bar{\lambda}(A)= & \sqrt{\frac{2 d \Lambda^{2}}{K_{0}}} \sqrt{\frac{A}{A_{\min }}\left(\frac{A}{A_{\min }}-1\right)}-\frac{A}{A_{\min }}\left(1+\frac{\Lambda^{2}}{K_{0}}\right) \\
& +\sqrt{\frac{2 K_{0}}{d \Lambda^{2}}}\left[\frac{A}{A_{\min }}\left(\frac{A}{A_{\min }}-1\right)\right]^{3 / 2}(\Delta j)^{2} .
\end{aligned}
$$


The integral over $\Delta j=j-j(A)$ goes along the imaginary axis and thus converges. For $F(A)$ we obtain

$$
F(A)=\sqrt{2 d \Lambda^{2} K_{0}} \sqrt{A\left(A-A_{\min }\right)}-A\left(K_{0}+\Lambda^{2}\right)+\frac{3}{4} \log \left[A\left(A-A_{\min }\right)\right]+\text { const. }
$$

According to the definition (5.1) of the string susceptibility index, we expect

$$
F(A)=\text { regular }+\left(2-\gamma_{\text {str }}\right) \log \frac{A}{A_{\min }}
$$

for $A \gg A_{\min }$. Comparing with (5.4), this determines $\gamma_{\mathrm{str}}=1 / 2$. It can be shown [24] that the one-loop correction contributes only to the regular part of $F(A)$ and does not change the singular part that gives $\gamma_{\text {str }}=1 / 2$. This value can be exact because it is linked only to the emergence of the square-root singularity which is not changed by higher orders.

We are to compare the mean-field result for $\gamma_{\text {str }}$ with the one-loop computation of (5.1) about the classical vacuum which is almost trivially done by changing $\rho \rightarrow A \rho$ and gives

$$
(5.1) \propto A^{-1} \mathrm{e}^{(d-2) \Lambda^{2} A / 2}
$$

resulting in $\gamma_{\mathrm{str}}=1$. We can compare it with the formula of the $d \rightarrow-\infty$ expansion [34] generalized to an arbitrary genus in [35, 36]. Since we deal with the worldsheet having topology of a cylinder which has two boundaries, its Euler character equals 0 like for a torus. This explains why there is no $d$-dependence of $\gamma_{\text {str }}$. We have got $\gamma_{\text {str }}=1$ rather than $\gamma_{\text {str }}=2$ as in $[35,36]$ because we deal with an open rather than a closed string.

The discrepancy between the obtained mean-field value $\gamma_{\text {str }}=1 / 2$ and the perturbative value $\gamma_{\text {str }}=1$ is due to the fact that the vacua are different. The former applies for $2<d<26$, while the latter applies for $d<2$.

\section{$6 \quad$ Fluctuations about the mean-field}

The instability of the effective action for $d>26$ implies that we cannot straightforwardly make a systematic $1 / d$ expansion as $d \rightarrow+\infty$. This is in contrast to the $d \rightarrow-\infty$ limit which comes along with the usual perturbative expansion because the vacuum is then just classical. The usual semiclassical expansion as $d \rightarrow-\infty$ cannot be extended to $d>2$ because the vacuum states are different for $d<2$ and $d>2$.

To go beyond the mean field for $2<d<26$, we define the partition function

$$
Z[h]=\int \mathcal{D} \rho \mathrm{e}^{-S_{\text {ind }} / h}
$$

with $S_{\text {ind }}$ given by eq. (4.14). Here we have introduced an additional parameter $h$ to control the "semiclassical" expansion about the mean field which plays the role of a "classical" vacuum. This procedure makes sense of the change $d \rightarrow d / h$ for the number of the $X$-fields and simultaneously $2 \rightarrow 2 / h$ for the number of the ghost fields. Diagrammatically, this mean field corresponds to summing up bubbles of both matter and ghosts. The mean-field approximation is associated with $h \rightarrow 0$, while the expansion about the mean field goes 
in $h$. Diagrams with $l$ loops of the field $\rho$ are then proportional to $h^{l}$. In reality $h=1$ but we can expect that the actual expansion parameter is $6 h /(26-d)$ like in the usual semiclassical expansion as $d \rightarrow-\infty$. Then the expansion can make sense for $d=4$.

The action in eq. (6.1) is given by (4.14). For the Pauli-Villars regularization the determinants are regularized by the ratio of massless to massive determinants [24]

$$
\left.\operatorname{det}(-\mathcal{O})\right|_{\text {reg }} \equiv \frac{\operatorname{det}(-\mathcal{O}) \operatorname{det}\left(-\mathcal{O}+2 M^{2}\right)}{\operatorname{det}\left(-\mathcal{O}+M^{2}\right)^{2}}
$$

so that

$$
\left.\operatorname{tr} \log (-\mathcal{O})\right|_{\text {reg }}=-\int_{0}^{\infty} \frac{\mathrm{d} \tau}{\tau} \operatorname{tr} \mathrm{e}^{\tau \mathcal{O}}\left(1-\mathrm{e}^{-\tau M^{2}}\right)^{2}
$$

is convergent. Here $M \rightarrow \infty$ is the regulator mass which is related to $\Lambda$ in the above equations by

$$
\Lambda^{2}=\frac{M^{2}}{2 \pi} \log 2
$$

For the proper-time regularization we have instead

$$
\left.\operatorname{det}(-\mathcal{O})\right|_{\text {reg }}=-\int_{a^{2}}^{\infty} \frac{\mathrm{d} \tau}{\tau} \operatorname{tr} \mathrm{e}^{\tau \mathcal{O}}, \quad \Lambda^{2}=\frac{1}{4 \pi a^{2}}
$$

We have added in (6.2) the ratio of the determinants for the masses $\sqrt{2} M$ and $M$ to cancel the logarithmic divergence at small $\tau$, because the Seeley expansion

$$
\left\langle\omega\left|\mathrm{e}^{\tau \mathcal{O}}\right| \omega\right\rangle=\frac{1}{4 \pi \tau} \frac{1}{\sqrt{\operatorname{det} \lambda}}+\frac{R}{24 \pi}+\ldots
$$

starts with the term $1 / \tau$. This is specific to the two-dimensional case.

The massive determinants in eq. (6.2) can also be represented as path integrals of the type

$$
\operatorname{det}\left(-\frac{\bar{\lambda}}{\rho} \partial^{2}+M^{2}\right)^{-d / 2}=\int \mathcal{D} X_{M}^{\mu} \mathrm{e}^{-\frac{K_{0}}{2} \int \mathrm{d}^{2} \omega\left(\bar{\lambda} \delta^{a b} \partial_{a} X_{M} \cdot \partial_{b} X_{M}+M^{2} \rho X_{M} \cdot X_{M}\right)}
$$

over the fields $X_{M}(\omega)$ with normal statistics or $Y_{M}(\omega)$ with ghost statistics and the double number of components. We can explicitly add these regulator fields to the action (4.12) to get

$$
\begin{aligned}
S= & K_{0}(1-\bar{\lambda}) \int \mathrm{d}^{2} \omega \rho+\frac{K_{0}}{2} \int \mathrm{d}^{2} \omega\left[\bar{\lambda} \partial_{a} X \cdot \partial_{a} X\right. \\
& \left.+\sum_{i=1}^{2}\left(\bar{\lambda} \partial_{a} Y_{M}^{(i)} \cdot \partial_{a} Y_{M}^{(i)}+M^{2} \rho\left(Y_{M}^{(i)}\right)^{2}\right)+\left(\bar{\lambda} \partial_{a} X_{\sqrt{2} M} \cdot \partial_{a} X_{\sqrt{2} M}+2 M^{2} \rho X_{\sqrt{2} M}^{2}\right)\right] .
\end{aligned}
$$

The path integral over the regulator fields generates the propagator

$$
\left\langle X_{M}^{\mu}(k) X_{M}^{\nu}(-k)\right\rangle=\frac{\delta^{\mu \nu}}{K_{0}\left(\bar{\lambda} k^{2}+M^{2} \bar{\rho}\right)}
$$


and the triple vertex of the $\delta \rho X_{M}^{\mu} X_{M}^{\nu}$ interaction

$$
\left\langle\delta \rho(-p) X_{M}^{\mu}(k+p) X_{M}^{\nu}(-k)\right\rangle_{\text {truncated }}=-K_{0} M^{2} \delta^{\mu \nu} .
$$

The latter vanishes for $M=0$ as it should owing to conformal invariance, but explicitly breaks it at nonzero $M$. Notice that path integration over all matter fields (both $X^{\mu}$ and the regulators) runs with a simple nonregularized measure. This makes it very convenient to derive (regularized) Noether's currents and to calculate their anomalies.

An instructive exercise is how to compute the usual anomaly in the trace of the energymomentum tensor

$$
\left[T_{a}^{a}\right]_{\mathrm{mat}}=4 \pi K_{0}\left[1-\bar{\lambda}+\frac{1}{2}\left(\sum_{i=1,2} M^{2}\left(Y_{M}^{(i)}\right)^{2}+2 M^{2} X_{\sqrt{2} M}^{2}\right)\right] .
$$

Averaging (6.11) over the regulator fields, we obtain the diagrams depicted in figure 1, where the solid line corresponds to the propagator of the regulator fields $X_{\sqrt{2} M}$ or $Y_{M}$ while the wavy line corresponds to $\delta \rho$. We have explicitly in momentum space

Figure $1 \mathrm{a}=2 \pi \frac{d}{h} \int \frac{\mathrm{d}^{2} k}{(2 \pi)^{2}}\left(\frac{2 M^{2}}{\bar{\lambda} k^{2}+2 M^{2} \bar{\rho}}-\frac{2 M^{2}}{\bar{\lambda} k^{2}+M^{2} \bar{\rho}}\right)=-\frac{d}{\bar{\lambda} h} M^{2} \log 2$,

reproducing eq. (6.4), and

$$
\begin{aligned}
\text { Figure } 1 \mathrm{~b}= & 2 \pi \frac{d}{h} \int \frac{\mathrm{d}^{2} k}{(2 \pi)^{2}}\left[\frac{4 M^{4}}{\left(\bar{\lambda} k^{2}+2 M^{2} \bar{\rho}\right)\left(\bar{\lambda}(k-p)^{2}+2 M^{2} \bar{\rho}\right)}\right. \\
& \left.-\frac{2 M^{4}}{\left(\bar{\lambda} k^{2}+M^{2} \bar{\rho}\right)\left(\bar{\lambda}(k-p)^{2}+M^{2} \bar{\rho}\right)}\right] \equiv \frac{d}{12 \bar{\rho}^{2} h} G(p) \stackrel{p \rightarrow 0}{\longrightarrow} \frac{d}{12 \bar{\rho}^{2} h} p^{2},
\end{aligned}
$$

where

$$
G(p)=12\left(\frac{4 m^{4} \operatorname{arctanh} \frac{p \sqrt{p^{2}+8 m^{2}}}{p^{2}+4 m^{2}}}{p\left(p^{2}+8 m^{2}\right)}-\frac{2 m^{4} \operatorname{arctanh} \frac{p \sqrt{p^{2}+4 m^{2}}}{p^{2}+2 m^{2}}}{p\left(p^{2}+4 m^{2}\right)}\right) \stackrel{p \lll m}{=} p^{2}
$$

and for brevity we denoted

$$
m^{2}=M^{2} \overline{\bar{\rho}} .
$$

The effect of the diagram in figure $1 \mathrm{c}$ and the next orders is to complete the result to scalar curvature $R$ as is discussed in appendix A. Adding all diagrams and using eq. (6.4), we obtain for the contribution from matter

$$
\left\langle\left[T_{a}^{a}\right]_{\mathrm{mat}}\right\rangle=4 \pi\left(K_{0}(1-\bar{\lambda})-\frac{d}{2 \bar{\lambda}} \Lambda^{2}\right)+\frac{d}{12} R .
$$

It still remains to compute the contribution of the ghost determinant which we also regularize by the Pauli-Villars regularization

$$
\left.\operatorname{det}\left(\left[-\mathcal{O}_{\mathrm{gh}}\right]_{b}^{a}\right)\right|_{\mathrm{reg}}=\frac{\operatorname{det}\left(\left[-\mathcal{O}_{\mathrm{gh}}\right]_{b}^{a}\right) \operatorname{det}\left(\left[-\mathcal{O}_{\mathrm{gh}}\right]_{b}^{a}+2 M^{2} \delta_{b}^{a}\right)}{\operatorname{det}\left(\left[-\mathcal{O}_{\mathrm{gh}}\right]_{b}^{a}+M^{2} \delta_{b}^{a}\right)^{2}} .
$$




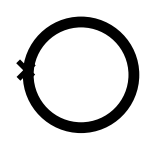

a)

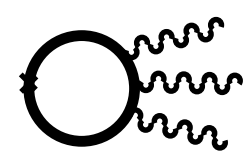

d)

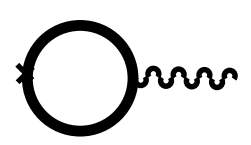

b)

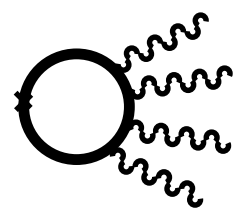

e)

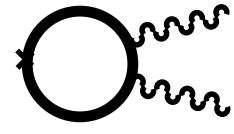

c)

Figure 1. Diagrams contributing to the average of (6.11) or (7.2) over the regulator fields.

The computation of the contribution from ghosts is pretty much similar to the one [25-27] for the perturbative vacuum and adding it with (6.16) we obtain for the trace of the total energy-momentum tensor (matter plus ghosts)

$$
\left\langle T_{a}^{a}\right\rangle \equiv\left\langle\left(\left[T_{a}^{a}\right]_{\mathrm{mat}}+\left[T_{a}^{a}\right]_{\mathrm{gh}}\right)\right\rangle=4 \pi\left[K_{0}(1-\bar{\lambda})-\left(\frac{d}{2 \bar{\lambda}}-1\right) \Lambda^{2}\right]+\frac{d-26}{12} R,
$$

which is the same as $\delta / \delta \rho$ acting on (4.15). The average in this formula is over the matter and ghost fields but not over $\rho$ which plays the role of an external field.

For $\bar{\lambda}$ given by eq. (2.9a) the divergent term vanishes, so we reproduce the usual conformal anomaly. The reason is that we have essentially made a one-loop calculation for the Polyakov-like action (4.12) with a constant fiducial metric $\hat{\rho}_{a b}=\bar{\rho} \delta_{a b}$ and the result coincides with the one about the classical vacuum because of the background independence.

\section{Computation of the central charge}

If $\rho_{a b}$ is considered as a classical background metric, only matter and ghosts contribute to the central charge of the Virasoro algebra which equals $d-26$ like in eq. (6.18). Then the conformal anomaly vanishes only in $d=26$ (the critical dimension) which reproduces the result of the old canonical quantization. We shall now see how this is modified when quantum fluctuations of $\rho_{a b}$ are taken into account in the mean-field approximation.

For this purpose let us compute the correlator of the two $z z$-components of the energymomentum tensor

$$
T_{z z} \equiv T(z)=T_{\mathrm{mat}}(z)+T_{\mathrm{gh}}(z)
$$

Classically, the $X$-field does not interact, as is already pointed out, with the metric $\rho$ in the conformal gauge because of conformal invariance. Like in the previous section we shall make use of the Pauli-Villars regularization, where $T_{\text {mat }}(z)$ explicitly depends on the regularizing fields as

$$
T_{\mathrm{mat}}(z)=2 \pi K_{0} \bar{\lambda}\left(\partial_{z} X \cdot \partial_{z} X+\partial_{z} X_{\sqrt{2} M} \cdot \partial_{z} X_{\sqrt{2} M}+\sum_{i=1}^{2} \partial_{z} Y_{M}^{(i)} \cdot \partial_{z} Y_{M}^{(i)}\right)
$$




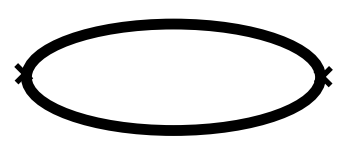

a)

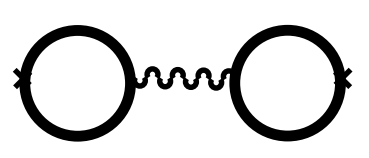

b)

Figure 2. Diagrams contributing to the correlator $\langle T(z) T(0)\rangle$ in the mean-field approximation.

The diagrams contributing to the correlator $\langle T(z) T(0)\rangle$ in the mean-field approximation are depicted in figure 2, where the solid line corresponds to the propagator of the field $X$ (and its regulators $X_{\sqrt{2} M}$ and $Y_{M}$ ) or the ghosts (and their regulators), while the wavy line corresponds to the propagator of $\delta \rho$

$$
\langle\delta \rho(-k) \delta \rho(k)\rangle=\frac{48 \pi h}{(26-d) k^{2}}, \quad\langle\delta \rho(z) \delta \rho(0)\rangle=-\frac{12 h}{(26-d)} \log (z \bar{z}) .
$$

To each closed line there is associated a factor of $(d-26) / h$ coming from summation over the matter and ghosts like in eq. (6.18).

The diagram in figure $2 \mathrm{a}$ (which have a combinatorial factor of 2 ) gives the usual result

$$
\langle T(z) T(0)\rangle_{a)}=\frac{d-26}{2 h z^{4}}
$$

associated with the central charges of free fields: $d$ for matter and 26 for ghosts, whose difference vanishes only in the critical dimension $d=26$. Only massless fields contribute to the most singular as $z \rightarrow 0$ part of the correlator shown in eq. (7.4) via the propagator

$$
\left\langle X_{q}^{\mu}(z) X_{q}^{\nu}(0)\right\rangle=-\frac{1}{4 \pi K_{0} \bar{\lambda}} \delta^{\mu \nu} \log (z \bar{z}) .
$$

The diagram in figure $2 \mathrm{~b}$ is usually associated with the next order of the perturbative expansion about the classical vacuum because it has two loops, but in the mean-field approximation it has to be considered together with the diagram in figure 2a since both are of the same order in $h$. We shall return soon to the discussion of this issue. Every of the two closed loops in the diagram in figure $2 \mathrm{~b}$ involves the momentum-space integral

$2 \pi \int \frac{\mathrm{d}^{2} k}{(2 \pi)^{2}} k_{z}\left(k_{z}-p_{z}\right)\left\{\frac{2 M^{2}}{\left(k^{2}+2 M^{2}\right)\left[(k-p)^{2}+2 M^{2}\right]}-\frac{2 M^{2}}{\left(k^{2}+M^{2}\right)\left[(k-p)^{2}+M^{2}\right]}\right\}=\frac{p_{z}^{2}}{12}$,

where we have absorbed the ratio $\bar{\rho} / \bar{\lambda}$ into $M^{2}$ for simplicity. The power counting predicts a quadratically divergent term like $M^{2} \rho_{z z}$ in the integral (7.6), but it vanishes in the conformal gauge.

Each of the two closed lines is associated ether with matter (the factor of $d$ ) of ghosts (the fector of -26). Multiplying the contribution of the two loops by the propagator (7.3), we find for the diagram in figure $2 \mathrm{~b}$

$$
\langle T(z) T(0)\rangle_{b)}=\frac{(d-26)}{12 h} \frac{12 h}{(26-d)} \frac{(d-26)}{12 h} \frac{6}{z^{4}}=-\frac{d-26}{2 h z^{4}} .
$$


Notice this result is pure anomalous: it comes entirely from the regulator fields but $M$ has canceled. Both diagrams in figure 2 give a "classical" (i.e. saddle-point) contribution from the viewpoint of the mean field. Adding (7.4) and (7.7), we obtain zero value of the total central charge in the mean-field approximation.

The fact that the total central charge of the bosonic string is always zero in the meanfield approximation, independently on the number of the target-space dimensions $d$, is remarkable. Thus it always reminds the string in the critical dimension $d=26$.

A very similar situation occurs in the Polchinski-Strominger approach [10] to the effective string theory, where the Alvarez-Arvis ground-state energy (same as (2.10) for $\bar{\lambda}=1$ ) was obtained from the requirement of vanishing the central charge at large $\beta$ to order $1 / \beta[10], 1 / \beta^{3}[16]$ and $1 / \beta^{5}[17]$. The mean-field approximation we used apparently sums up bubble graphs to all orders in $1 / \beta$ and explicitly results in the Alvarez-Arvis formula.

\section{8 "Semiclassical" correction to the mean field}

Let us consider a "semiclassical" correction to the mean-field approximation which comes from averaging over fluctuations of $\rho$ about $\bar{\rho}$.

Integrating over the matter and ghost fields (including their regulators), we obtain the following induced action for the field $\delta \rho$ to quadratic order in $\delta \rho$ :

$$
S_{\text {ind }}^{(2)}=\frac{(26-d)}{96 \pi h} \int \frac{\mathrm{d}^{2} p}{(2 \pi)^{2}} \delta \rho(-p) G(p) \delta \rho(p)
$$

with $G(p)$ given by eq. (6.14).

This is not the end of the story because there are diagrams with three, four, etc. $\delta \rho$ 's in (4.14), whose contributions we denote as $S_{\text {ind }}^{(3)}, S_{\text {ind }}^{(4)}$, etc. As is explicitly demonstrated in appendix $\mathrm{A}$, it is convenient to introduce instead of $\delta \rho$ another variable $\varphi$ by

$$
\rho(z)=\bar{\rho} \mathrm{e}^{\varphi(z)}, \quad \delta \rho(z)=\bar{\rho}\left(\mathrm{e}^{\varphi(z)}-1\right)
$$

and to expand in $\varphi$. Then the terms higher than quadratic order in $\varphi$ are mutually canceled in the sum

$$
S_{\text {ind }}=S_{\text {ind }}^{(2)}+S_{\text {ind }}^{(3)}+\ldots=\frac{(26-d)}{96 \pi h} \int \frac{\mathrm{d}^{2} p}{(2 \pi)^{2}} \varphi(-p) G(p) \varphi(p)+\mathcal{O}\left(\varphi^{3}\right)
$$

in the IR limit where all variables $p_{i}$ 's obey $p_{i} p_{j} \ll M^{2} \bar{\rho}$, so the induced action (8.3) reproduces the usual effective action for smooth $\varphi(z)$. However, we consider below explicitly the case of four $\varphi$ 's, where two momenta are small, $p_{i}^{2} \ll M^{2} \bar{\rho}$, but two other momenta are large, $p_{i}^{2} \sim M^{2} \bar{\rho}$. There is no reason to expect the cancellation in this case.

The effective action describes "slow" fluctuations of $\varphi$ with $p^{2} \ll M^{2} \bar{\rho}$ and emerges after averaging over "fast" fluctuations with $p^{2} \sim M^{2} \bar{\rho}$. The quadratic part of the effective action gets then contribution from averaging higher terms in the induced action (which are generically nonlocal), so we write it in the spirit of DDK (a good review is [37]) as

$$
S_{\text {eff }}=\frac{1}{16 \pi b^{2}} \int \mathrm{d}^{2} z \partial_{a} \varphi \partial_{a} \varphi+\mathcal{O}\left(M^{-2}\right)
$$




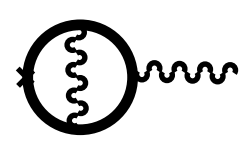

a)

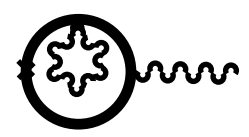

d)

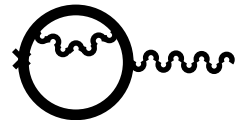

b)

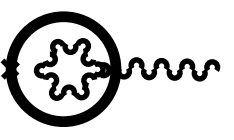

e)

Figure 3. Diagrams associated with the next order correction to the mean field for $T_{z z}$. The combinatorial factors are $\left.a)+2 b)-2 c)-d)+\frac{1}{2} e\right)$.

with a certain constant $b^{2}$. The difference between the induced action (8.3) and the effective action (8.4) will show up when virtual momenta of the propagator $\langle\varphi(-p) \varphi(p)\rangle$ in diagrams, which emerge after averaging over $\varphi$, are large: $p^{2} \gtrsim M^{2} \bar{\rho}$. Hence the higher order in $\varphi$ terms in eq. (8.3) may and will, as we see below, then play an important role. The reason why they survive is, roughly speaking, a quadratic divergence of the involved integrals. These terms are however subordinated in $h$ because $\langle\varphi(-p) \varphi(p)\rangle \propto h$ owing to eq. (7.3).

It is instructive to give yet another explanation why the higher terms can emerge. Let us consider $T_{z z}$ given by eqs. (7.1) and average (7.2) over the regulator fields with $\varphi$ playing again the role of an external field. The result is given by the diagrams in figure 1 whose analytic expressions are listed in eqs. (A.7)-(A.11) of appedix A, where it is explicitly shown the cancellation of higher than quadratic terms when all momenta squared of external lines are small (i.e. $p_{i}^{2} \ll M^{2} \bar{\rho}$ ). I do not see again any reason to expect such a cancellation for momenta of the order of $M^{2} \bar{\rho}$, so counterparts of the higher terms in eq. (8.3) may emerge.

The result of the averaging over the regulators will not be yet the energy-momentum (pseudo)tensor because the averages in the path-integral language are associated with $T$ products in the operator language. To obtain a genuine $T_{z z}$, we have to normal order the operators $\varphi$ which produces additional terms like the diagrams in figure 3 coming from normal ordering in $\varphi^{4}$. We thus write

$$
\boldsymbol{T}_{z z}^{\varphi}=\frac{1}{2}\left(\frac{1}{2 b^{2}}: \partial_{z} \varphi \partial_{z} \varphi:-Q \partial_{z}^{2} \boldsymbol{\varphi}\right)+\mathcal{O}\left(\varphi^{3}\right)
$$

again in the spirit of DDK.

One more source of the nonlinearity is the well-known fact that the norm of $\varphi$ is nonlinear

$$
\|\delta \varphi\|^{2}=\int \mathrm{d}^{2} z \rho(z)(\delta \varphi(z))^{2} .
$$

We can adopt the philosophy of DDK and replace the path integral over $\varphi$ with the non- 
linear norm (8.6) by the path integral over the field $\varphi_{0}$ with a linear one

$$
\left\|\delta \varphi_{0}\right\|^{2}=\int \mathrm{d}^{2} z \bar{\rho}\left(\delta \varphi_{0}(z)\right)^{2}
$$

by introducing the Jacobian for the transformation from $\varphi$ to $\varphi_{0}$. It has again the form of (the exponential of) the action (8.3) and simply changes its coefficients. We shall therefore replace in eq. (8.3)

$$
\frac{26-d}{6 h} \Rightarrow \frac{1}{b_{0}^{2}}, \quad \frac{1}{b_{0}^{2}}=\frac{26-d}{6 h}+\mathcal{O}(1) .
$$

The difference between this $b_{0}^{2}$ and $b^{2}$ in eq. (8.4) comes to order $h$ from the diagrams with one propagator which are computed below.

My last comment before proceeding with the computations is that the propagator $\langle\varphi(-p) \varphi(p)\rangle$ behaves as $1 / p^{2}$ for small $p^{2}$, so one might expect therefore logarithmic IR divergences, associated with this behavior, which would spoil conformal invariance. However, the low-momentum effective action is quadratic in the variable $\varphi$ as is already mentioned (and demonstrated by explicit computations in appendix A), so the divergences are expected to cancel each other because the induced action coincides with the effective action in the IR domain. We shall see in explicit computations this is indeed the case. The remaining contribution to be calculated will come from virtual momenta squared of the order of the cutoff: $k^{2} \sim M^{2} \bar{\rho}$. I believe this is a heuristic proof of the theorem about the cancellation of the IR divergences.

\subsection{Correction to $T_{z z}$}

The diagrams of the next to the leading order in $h$ which describe "quantum" corrections to the mean-field approximation for $T_{z z}$ are depicted in figure 3. Their analytic expressions are listed in eqs. (B.1)-(B.5) of appendix B.

Every individual diagram has an IR divergence coming from the $\varphi-\varphi$ propagator, but it has indeed canceled in the sum as anticipated. Actually the cancellation happens for the $\operatorname{sum} a)+2 b)-2 c$ ) because $d)=\frac{1}{2} e$ ) so only the diagrams in figure $3 \mathrm{a}, 3 \mathrm{~b}$ and $3 \mathrm{c}$ contribute resulting in

$$
\text { a) }+2 b)-2 c)=-\frac{13 p_{z}^{2}}{288} \text {. }
$$

It is instructive to present the result in the DDK form

$$
T_{z z}^{\varphi}=\frac{1}{2}\left(\frac{1}{2 b^{2}} \partial_{z} \varphi \partial_{z} \varphi-Q \partial_{z}^{2} \varphi\right)
$$

Multiplying (8.9) by the normalizations of the propagator (7.3) and of the integrals and summing with the leading-order diagrams in figure 1 , we obtain for the coefficient $Q$ in eq. (8.5)

$$
Q=\frac{q_{0}}{b_{0}^{2}}-\frac{13}{6}+\mathcal{O}(h)
$$

where $b_{0}^{2}$ is defined in eq. (8.8). 


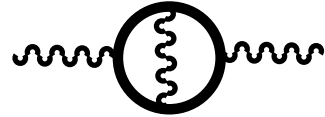

a)

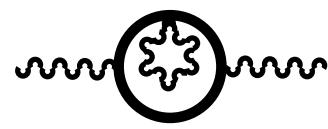

d)

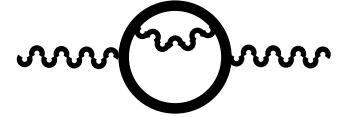

b)

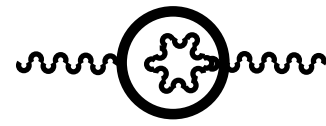

e)

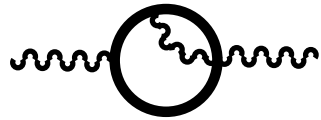

c)

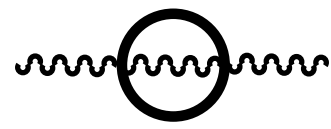

f)

Figure 4. Diagrams associated with the next order correction to the mean field for $S_{\text {eff }}$ The combinatorial factors are $a)+2 b)-4 c)-d)+e)+f)$.

An analogous direct computation of the quadratic in $\varphi$ term in eq. (8.5) is a bit more tedious and involves 12 diagrams: 7 of which are new, while the contribution of the sum of remaining 5 diagrams is like $\frac{1}{2} Q \partial^{2} \varphi^{2}$. The integrals involve two external momenta, which complicates their computation.

\subsection{Correction to $S_{\text {eff }}$}

The diagrams of the next to the leading order in $h$ which describe "quantum" corrections to the mean-field approximation for $S_{\text {eff }}$ are depicted in figure 4. Their analytic expressions are listed in eqs. (B.12)-(B.17) of appendix B. Every individual diagram has again an IR divergence coming from the $\varphi-\varphi$ propagator, but it has canceled in the sum as anticipated. Actually the cancellation happens for the sum $a)+2 b)-4 c)+f$ ) because $d$ ) $=e$ ), so only the diagrams in figure $4 \mathrm{a}, 4 \mathrm{~b}, 4 \mathrm{c}$ and $4 \mathrm{f}$ contribute. Accounting for combinatorial factors, we obtain

$$
\text { a) }+2 b)-4 c)+f)=-\frac{5 p^{2}}{48} .
$$

Multiplying (8.12) by the normalization of the propagator (7.3) and of the integrals, accounting for ghosts and summing with the mean-field result, we obtain for the coupling constant in the effective action (8.4)

$$
\frac{1}{b^{2}}=\frac{1}{b_{0}^{2}}-5+\mathcal{O}(h)
$$

\subsection{Remark on the universality}

In the above computations of $T_{z z}$ and $S_{\text {eff }}$ we substituted $G(p)$ in eq. (8.3) by $p^{2}$ because otherwise the computation is hopeless. A question arises as to whether this affects the results because characteristic virtual momenta squared in the diagrams are $\sim M^{2} \bar{\rho}$.

It is possible to verify that by changing the regularization procedure (6.3) to

$$
\left.\operatorname{tr} \log (-\mathcal{O})\right|_{\text {reg }}=-\int_{0}^{\infty} \frac{\mathrm{d} \tau}{\tau} \operatorname{tr} \mathrm{e}^{\tau \mathcal{O}}\left(1-\mathrm{e}^{-\tau M^{2}}\right)^{N} .
$$


Such a modification of the Pauli-Villars regularization is discussed in [24]. The regularization (8.14) involves $N$ Pauli-Villars regulators with masses $\sqrt{n} M(n=1, \ldots, N)$ which complicates the computation. It can be shown however that the results (8.11), (8.13) do not change which is an argument in favor of their universality.

\section{Discussion}

The main result of this Paper is that a quantization of the effective string about the mean-field ground state works in $2<d<26$. The mean-field approximation corresponds to conformal field theory with the central charge vanishing for any $d$, resulting in the Alvarez-Arvis ground-state energy and complimenting the Polchinski-Strominger approach. A "semiclassical" expansion about the mean field can be treated adopting the philosophy of DDK.

The difference from DDK is that our $\varphi$ is massless as a consequence of the minimization at the mean-field saddle point. The massless $\varphi$ is thus a consequence of the nonperturbative mean-field ground state for $2<d<26$ in contrast to the usual perturbative one for $d<2$. This may lead to infrared logarithms which would spoil conformal invariance when accounting for fluctuations about the mean field, but we argued they have to cancel because the low-momentum (or effective) action is quadratic in $\varphi$. This cancellation is explicitly shown to the lowest order of the "semiclassical" expansion about the mean field. Thus, we expect that conformal invariance should be maintained order by order of the expansion.

The explicit computation shows the (induced) action governing fluctuations about the mean field is however not quadratic in $\varphi$, while only its low-momentum limit - the effective action - is quadratic. The reason for that is, roughly speaking, quadratic divergences of the involved integrals. Using the Pauli-Villars regularization, I have shown how to systematically treat the induced action (4.14) and to deal with these higher order terms without assuming that $\varphi$ is smooth and the determinants are approximated by the conformal anomaly. Their emergence may influence the results and deserve further investigation.

The most interesting question is what would be the spectrum of the Nambu-Goto string beyond the mean-field approximation. In particular, whether the universal correction to the Alvarez-Arvis spectrum at the order $1 / \beta^{5}$ (see [12] and references therein) is reproduced in the "semiclassical" expansion about the mean field at one loop. This issue will be considered elsewhere.

\section{Acknowledgments}

I am grateful to Jan Ambjørn for sharing his insight into Strings.

\section{A Leading-order explicit computations}

To the leading order in $h$ we consider $\delta \rho($ or $\varphi$ ) as an external field over which we shall average to next orders. 


\section{A.1 $T_{a}^{a}$}

The contribution of diagrams in figure 1 to $T_{a}^{a}$ from the regulators read

$$
\begin{aligned}
& a)=\int \frac{\mathrm{d}^{2} k}{(2 \pi)^{2}}\left\{\frac{2 M^{2}}{\left(k^{2}+2 M^{2}\right)}-\frac{2 M^{2}}{\left(k^{2}+M^{2}\right]}\right\}=-\frac{M^{2}}{2 \pi} \log 2, \\
& b)=\int \frac{\mathrm{d}^{2} k}{(2 \pi)^{2}}\left\{\frac{4 M^{4}}{\left(k^{2}+2 M^{2}\right)\left[(k-p)^{2}+2 M^{2}\right]}-\frac{2 M^{4}}{\left(k^{2}+M^{2}\right)\left[(k-p)^{2}+M^{2}\right]}\right\} \stackrel{M \rightarrow \infty}{=} \frac{p^{2}}{24 \pi}, \\
& c)=\int \frac{\mathrm{d}^{2} k}{(2 \pi)^{2}}\left\{\frac{8 M^{6}}{\left(k^{2}+2 M^{2}\right)\left[(k-p)^{2}+2 M^{2}\right]\left[(k-p-q)^{2}+2 M^{2}\right]}\right. \\
& \left.-\frac{2 M^{6}}{\left(k^{2}+M^{2}\right)\left[(k-p)^{2}+M^{2}\right]\left[(k-p-q)^{2}+M^{2}\right]}\right\} \stackrel{M \rightarrow \infty}{=} \frac{p^{2}+q^{2}+p q}{24 \pi}, \\
& d)=\int \frac{\mathrm{d}^{2} k}{(2 \pi)^{2}}\left\{\frac{16 M^{8}}{\left(k^{2}+2 M^{2}\right)\left[(k-p)^{2}+2 M^{2}\right]\left[(k-p-q)^{2}+2 M^{2}\right]\left[(k-p-q-r)^{2}+2 M^{2}\right]}\right. \\
& \left.-\frac{2 M^{8}}{\left(k^{2}+M^{2}\right)\left[(k-p)^{2}+M^{2}\right]\left[(k-p-q)^{2}+M^{2}\right]\left[(k-p-q-r)^{2}+M^{2}\right]}\right\} \\
& \stackrel{M \rightarrow \infty}{=} \frac{3 p^{2}+4 q^{2}+3 r^{2}+4 p q+2 p r+4 q r}{80 \pi} \\
& e)=\int \frac{\mathrm{d}^{2} k}{(2 \pi)^{2}}\left\{\frac{32 M^{10}}{\left(k^{2}+2 M^{2}\right) \cdots\left[(k-p-q-r)^{2}+2 M^{2}\right]\left[(k-p-q-r-t)^{2}+2 M^{2}\right]}\right. \\
& \left.-\frac{2 M^{10}}{\left(k^{2}+M^{2}\right)\left[(k-p)^{2}+M^{2}\right] \cdots\left[(k-p-q-r)^{2}+M^{2}\right]\left[(k-p-q-r-t)^{2}+M^{2}\right]}\right\} \\
& \stackrel{M \rightarrow \infty}{=} \frac{2 p^{2}+3 q^{2}+3 r^{2}+2 t^{2}+3 p q+2 p r+p t+4 q r+2 q t+3 r t}{60 \pi} .
\end{aligned}
$$

Multiplying each wavy line by $-\delta \rho$, passing to the coordinate space and summing up the contributions of the diagrams in figure 1 with these of ghosts, we find

$$
\begin{aligned}
\left\langle T_{a}^{a}\right\rangle= & \frac{26-d}{12 h}\left[\partial^{2} \delta \rho-\left(2 \delta \rho \partial^{2} \delta \rho+\partial_{a} \delta \rho \partial_{a} \delta \rho\right)+3\left(\delta \rho^{2} \partial^{2} \delta \rho+\delta \rho \partial_{a} \delta \rho \partial_{a} \delta \rho\right)\right. \\
& \left.-\left(4 \delta \rho^{3} \partial^{2} \delta \rho+6 \delta \rho^{2} \partial_{a} \delta \rho \partial_{a} \delta \rho\right)\right]+\mathcal{O}\left(\delta \rho^{5}\right) \\
= & \frac{26-d}{12 h}\left(1-\varphi+\frac{1}{2} \varphi^{2}-\frac{1}{6} \varphi^{3}\right) \partial^{2} \varphi+\mathcal{O}\left(\varphi^{5}\right),
\end{aligned}
$$

where we have used (8.2) and expanded in $\varphi$. We have thus reproduced eq. (6.18) to this order.

In eq. (A.6) we simply subtracted 26 from $d$ to account for the ghost contribution because the contribution of diagrams which emerge from ghost and the regulators of ghosts are identical for the mean-field and perturbative vacua just as it is for the matter fields and regulators. The same applies below for $T_{z z}$. 


\section{A.2 $T_{z z}$}

The analogous contribution of diagrams in figure 1 to $T_{z z}$ read

$a)=\int \frac{\mathrm{d}^{2} k}{(2 \pi)^{2}}\left\{\frac{k_{z}^{2}}{\left(k^{2}+2 M^{2}\right)}-\frac{k_{z}^{2}}{\left(k^{2}+M^{2}\right)}\right\}=0$,

$b)=\int \frac{\mathrm{d}^{2} k}{(2 \pi)^{2}}\left\{\frac{2 M^{2} k_{z}\left(k_{z}-p_{z}\right)}{\left(k^{2}+2 M^{2}\right)\left[(k-p)^{2}+2 M^{2}\right]}-\frac{2 M^{2} k_{z}\left(k_{z}-p_{z}\right)}{\left(k^{2}+M^{2}\right)\left[(k-p)^{2}+M^{2}\right]}\right\} \stackrel{M \rightarrow \infty}{=} \frac{p_{z}^{2}}{24 \pi}$,

$c)=\int \frac{\mathrm{d}^{2} k}{(2 \pi)^{2}}\left\{\frac{4 M^{4} k_{z}\left(k_{z}-p_{z}-q_{z}\right)}{\left(k^{2}+2 M^{2}\right)\left[(k-p)^{2}+2 M^{2}\right]\left[(k-p-q)^{2}+2 M^{2}\right]}\right.$

$$
\left.-\frac{2 M^{4} k_{z}\left(k_{z}-p_{z}-q_{z}\right)}{\left(k^{2}+M^{2}\right)\left[(k-p)^{2}+M^{2}\right]\left[(k-p-q)^{2}+M^{2}\right]}\right\} \stackrel{M \rightarrow \infty}{=} \frac{p_{z}^{2}+q_{z}^{2}+3 p_{z} q_{z}}{48 \pi},
$$

$d)=\int \frac{\mathrm{d}^{2} k}{(2 \pi)^{2}}\left\{\frac{8 M^{6} k_{z}\left(k_{z}-p_{z}-q_{z}-r_{z}\right)}{\left(k^{2}+2 M^{2}\right)\left[(k-p)^{2}+2 M^{2}\right]\left[(k-p-q)^{2}+2 M^{2}\right]\left[(k-p-q-r)^{2}+2 M^{2}\right]}\right.$

$$
\left.-\frac{2 M^{6} k_{z}\left(k_{z}-p_{z}-q_{z}-r_{z}\right)}{\left(k^{2}+M^{2}\right)\left[(k-p)^{2}+M^{2}\right]\left[(k-p-q)^{2}+M^{2}\right]\left[(k-p-q-r)^{2}+M^{2}\right]}\right\}
$$

$M \stackrel{M}{=} \frac{3 p_{z}^{2}+4 q_{z}^{2}+3 r_{z}^{2}+9 p_{z} q_{z}+12 p_{z} r_{z}+9 q_{z} r_{z}}{240 \pi}$,

$e)=\int \frac{\mathrm{d}^{2} k}{(2 \pi)^{2}}\left\{\frac{16 M^{8} k_{z}\left(k_{z}-p_{z}-q_{z}-r_{z}-t_{z}\right)}{\left(k^{2}+2 M^{2}\right) \cdots\left[(k-p-q-r)^{2}+2 M^{2}\right]\left[(k-p-q-r-t)^{2}+2 M^{2}\right]}\right.$

$$
\left.-\frac{2 M^{8} k_{z}\left(k_{z}-p_{z}-q_{z}-r_{z}-t_{z}\right)}{\left(k^{2}+M^{2}\right)\left[(k-p)^{2}+M^{2}\right] \cdots\left[(k-p-q-r)^{2}+M^{2}\right]\left[(k-p-q-r-t)^{2}+M^{2}\right]}\right\}
$$

$\stackrel{M \rightarrow \infty}{=} \frac{2 p_{z}^{2}+3 q_{z}^{2}+3 r_{z}^{2}+2 t_{z}^{2}+6 p_{z} q_{z}+8 p_{z} r_{z}+10 p_{z} t_{z}+7 q_{z} r_{z}+8 q_{z} t_{z}+6 r_{z} t_{z}}{240 \pi}$.

Using (8.2), we analogously to eq. (A.6) obtain for $T_{z z}^{\varphi}$

$$
\begin{aligned}
\left\langle T_{z z}\right\rangle= & \frac{26-d}{12 h}\left[\partial_{z}^{2} \delta \rho-\left(\delta \rho \partial_{z}^{2} \delta \rho+\frac{3}{2} \partial_{z} \delta \rho \partial_{z} \delta \rho\right)+\left(\delta \rho^{2} \partial_{z}^{2} \delta \rho+3 \delta \rho \partial_{z} \delta \rho \partial_{z} \delta \rho\right)\right. \\
& \left.-\left(\delta \rho^{3} \partial_{z}^{2} \delta \rho+\frac{9}{2} \delta \rho^{2} \partial_{z} \delta \rho \partial_{z} \delta \rho\right)\right]+\mathcal{O}\left(\delta \rho^{5}\right) \\
= & \frac{26-d}{12 h}\left(\partial_{z}^{2} \varphi-\frac{1}{2} \partial_{z} \varphi \partial_{z} \varphi\right)+\mathcal{O}\left(\varphi^{5}\right)
\end{aligned}
$$

i.e. the free energy-momentum tensor to this order.

The reason why I presented in this appendix the explicit computations of $T_{a}^{a}$ and $T_{z z}$ is to emphasize that numerical factors are most important to get the free-theory results. The cancellation would no longer take place if these factors were changed due to induced interactions, as we shall immediately see in the next appendix. 


\section{B "Semiclassical" corrections}

\section{B.1 Contribution to $T_{z z}$}

The contributions of diagrams in figure 3 to $T_{z z}$ involve

$$
\begin{aligned}
a)= & \int \frac{\mathrm{d}^{2} k \mathrm{~d}^{2} q}{(2 \pi)^{2}}\left\{\frac{8 M^{6} k_{z}\left(k_{z}-p_{z}\right)}{(k-q)^{2}\left[(k-p)^{2}+2 M^{2}\right]\left[(q-p)^{2}+2 M^{2}\right]\left(k^{2}+2 M^{2}\right)\left(q^{2}+2 M^{2}\right)}\right. \\
& \left.-\frac{2 M^{6} k_{z}\left(k_{z}-p_{z}\right)}{(k-q)^{2}\left[(k-p)^{2}+M^{2}\right]\left[(q-p)^{2}+M^{2}\right]\left(k^{2}+M^{2}\right)\left(q^{2}+M^{2}\right)}\right\} \\
b= & \int \frac{\mathrm{d}^{2} k \mathrm{~d}^{2} q}{(2 \pi)^{2}}\left\{\frac{8 M^{6} k_{z}\left(k_{z}-p_{z}\right)}{(k-q)^{2}\left[(k-p)^{2}+2 M^{2}\right]\left(k^{2}+2 M^{2}\right)^{2}\left(q^{2}+2 M^{2}\right)}\right. \\
& \left.-\frac{2 M^{6} k_{z}\left(k_{z}-p_{z}\right)}{(k-q)^{2}\left[(k-p)^{2}+M^{2}\right]\left(k^{2}+M^{2}\right)^{2}\left(q^{2}+M^{2}\right)}\right\} \\
c)= & \int \frac{\mathrm{d}^{2} k \mathrm{~d}^{2} q}{(2 \pi)^{2}}\left\{\frac{4 M^{4} k_{z}\left(k_{z}-p_{z}\right)}{(k-q)^{2}\left[(k-p)^{2}+2 M^{2}\right]\left(k^{2}+2 M^{2}\right)\left(q^{2}+2 M^{2}\right)}\right. \\
d)= & \left.\int \frac{2 M^{4} k_{z}\left(k_{z}-p_{z}\right)}{(k-q)^{2}\left[(k-p)^{2}+M^{2}\right]\left(k^{2}+M^{2}\right)\left(q^{2}+M^{2}\right)}\right\} \\
& \int \frac{4 \mathrm{~d}^{2} q}{(2 \pi)^{2}}\left\{\frac{2 M_{z}^{4} k_{z}\left(k_{z}-p_{z}\right)}{q^{2}\left[(k-p)^{2}+2 M^{2}\right]\left(k^{2}+2 M^{2}\right)^{2}}-\frac{2 M^{2} k_{z}\left(k_{z}-p_{z}\right)}{q^{2}\left[(k-p)^{2}+M^{2}\right]\left(k^{2}+M^{2}\right)^{2}}\right\} \\
e)= & \int \frac{\mathrm{d}^{2} k \mathrm{~d}^{2} q}{(2 \pi)^{2}}\left\{\frac{2 M^{2} k_{z}\left(k_{z}-p_{z}\right)}{q^{2}\left[(k-p)^{2}+2 M^{2}\right]\left(k^{2}+2 M^{2}\right)}-\frac{2 M^{2}\left[(k-p)^{2}+M^{2}\right]\left(k^{2}+M^{2}\right)}{q^{2}}\right\}
\end{aligned}
$$

For the computation of integrals it is convenient to multiply a generic integral

$$
\int \frac{\mathrm{d}^{2} k}{(2 \pi)^{2}} k_{a}\left(k_{b}-p_{b}\right) f\left(k^{2}, p^{2}, k p\right)=f_{1}\left(p^{2}\right) g_{a b}+f_{2}\left(p^{2}\right) p_{a} p_{b}
$$

by the projector

$$
P^{a b}=2 \frac{p^{a} p^{b}}{p^{2}}-g^{a b}
$$

to get

$$
\int \frac{\mathrm{d}^{2} k}{(2 \pi)^{2}}\left(2 \frac{(k p)^{2}}{p^{2}}-k p-k^{2}\right) f\left(k^{2}, p^{2}, k p\right)=f_{2}\left(p^{2}\right) p^{2} .
$$

Then we have

$$
\int \frac{\mathrm{d}^{2} k}{(2 \pi)^{2}} k_{z}\left(k_{z}-p_{z}\right) f\left(k^{2}, p^{2}, k p\right)=f_{2}\left(p^{2}\right) p_{z}^{2} .
$$

This trick is implemented in the Mathematica program of appendix C, where the integrals are computed by first integrating over the two relative angles and then by the two absolute values of the virtual momenta.

Performing the computation by the Mathematica program in appendix $\mathrm{C}$ and accounting for combinatorial factors, we obtain

$$
\left.a)+2 b)-2 c)-d)+\frac{1}{2} e\right)=-\frac{13 p_{z}^{2}}{288} .
$$


Notice that $d)=\frac{1}{2} e$ ) so only the diagrams in figure 3a, 3b and 3c contribute. The infrared divergence coming from the $\varphi-\varphi$ propagator has indeed canceled in the sum, as anticipated.

Multiplying (B.10) by the normalization of the propagator (7.3) and of the integrals and summing the diagrams in figure 1 and figure 3 , we obtain

$$
T_{z z}^{\varphi}=\frac{26-d}{12 h}\left[\left(1-\frac{13 h}{26-d}\right) \partial_{z}^{2} \varphi-\frac{1}{2} \partial_{z} \varphi \partial_{z} \varphi\right]+\mathcal{O}(h) .
$$

\section{B.2 Contribution to $S_{\text {eff }}$}

The contributions of diagrams in figure 4 to $S_{\text {eff }}$ involve

$$
\begin{aligned}
& a)=\int \frac{\mathrm{d}^{2} k \mathrm{~d}^{2} q}{(2 \pi)^{2}}\left\{\frac{16 M^{8}}{(k-q)^{2}\left[(k-p)^{2}+2 M^{2}\right]\left[(q-p)^{2}+2 M^{2}\right]\left(k^{2}+2 M^{2}\right)\left(q^{2}+2 M^{2}\right)}\right. \\
& \left.-\frac{2 M^{8}}{(k-q)^{2}\left[(k-p)^{2}+M^{2}\right]\left[(q-p)^{2}+M^{2}\right]\left(k^{2}+M^{2}\right)\left(q^{2}+M^{2}\right)}\right\}, \\
& b)=\int \frac{\mathrm{d}^{2} k \mathrm{~d}^{2} q}{(2 \pi)^{2}}\left\{\frac{16 M^{8}}{(k-q)^{2}\left[(k-p)^{2}+2 M^{2}\right]\left(k^{2}+2 M^{2}\right)^{2}\left(q^{2}+2 M^{2}\right)}\right. \\
& \left.-\frac{2 M^{8}}{(k-q)^{2}\left[(k-p)^{2}+M^{2}\right]\left(k^{2}+M^{2}\right)^{2}\left(q^{2}+M^{2}\right)}\right\} \text {, } \\
& c)=\int \frac{\mathrm{d}^{2} k \mathrm{~d}^{2} q}{(2 \pi)^{2}}\left\{\frac{8 M^{6}}{(k-q)^{2}\left[(k-p)^{2}+2 M^{2}\right]\left(k^{2}+2 M^{2}\right)\left(q^{2}+2 M^{2}\right)}\right. \\
& \left.-\frac{2 M^{6}}{(k-q)^{2}\left[(k-p)^{2}+M^{2}\right]\left(k^{2}+M^{2}\right)\left(q^{2}+M^{2}\right)}\right\} \text {, } \\
& d)=\int \frac{\mathrm{d}^{2} k \mathrm{~d}^{2} q}{(2 \pi)^{2}}\left\{\frac{8 M^{6}}{q^{2}\left[(k-p)^{2}+2 M^{2}\right]\left(k^{2}+2 M^{2}\right)^{2}}-\frac{2 M^{6}}{q^{2}\left[(k-p)^{2}+M^{2}\right]\left(k^{2}+M^{2}\right)^{2}}\right\} \text {, } \\
& e)=\int \frac{\mathrm{d}^{2} k \mathrm{~d}^{2} q}{(2 \pi)^{2}}\left\{\frac{4 M^{4}}{q^{2}\left[(k-p)^{2}+2 M^{2}\right]\left(k^{2}+2 M^{2}\right)}-\frac{2 M^{4}}{q^{2}\left[(k-p)^{2}+M^{2}\right]\left(k^{2}+M^{2}\right)}\right\} \\
& f)=\int \frac{\mathrm{d}^{2} k \mathrm{~d}^{2} q}{(2 \pi)^{2}}\left\{\frac{4 M^{4}}{(k-q)^{2}\left[(k-p)^{2}+2 M^{2}\right]\left(q^{2}+2 M^{2}\right)}\right. \\
& \left.-\frac{2 M^{4}}{(k-q)^{2}\left[(k-p)^{2}+M^{2}\right]\left(q^{2}+M^{2}\right)}\right\} \text {. }
\end{aligned}
$$

Performing the computation by the Mathematica program of appendix $\mathrm{C}$ and accounting for combinatorial factors, we obtain

$$
a)+2 b)-4 c)-d)+e)+f)=-\frac{5 p^{2}}{48} .
$$

Notice that again $d)=e$ ) so only the diagrams in figure $4 \mathrm{a}, 4 \mathrm{~b}, 4 \mathrm{c}$ and $4 \mathrm{f}$ contribute. The infrared divergence coming from the $\varphi-\varphi$ propagator has indeed canceled in the sum, as anticipated. 
Multiplying (B.18) by the normalization of the propagator (7.3) and of the integrals, accounting for ghosts and summing with the mean-field result, we obtain

$$
S_{\text {eff }}=\frac{26-d}{96 \pi h}\left(1-\frac{30 h}{26-d}\right) \int \mathrm{d}^{2} z \partial_{a} \varphi \partial_{a} \varphi+\mathcal{O}(h) .
$$

\section{Mathematica programs}

\section{C.1 Program for computing diagrams in figure 3 that results in (8.9)}

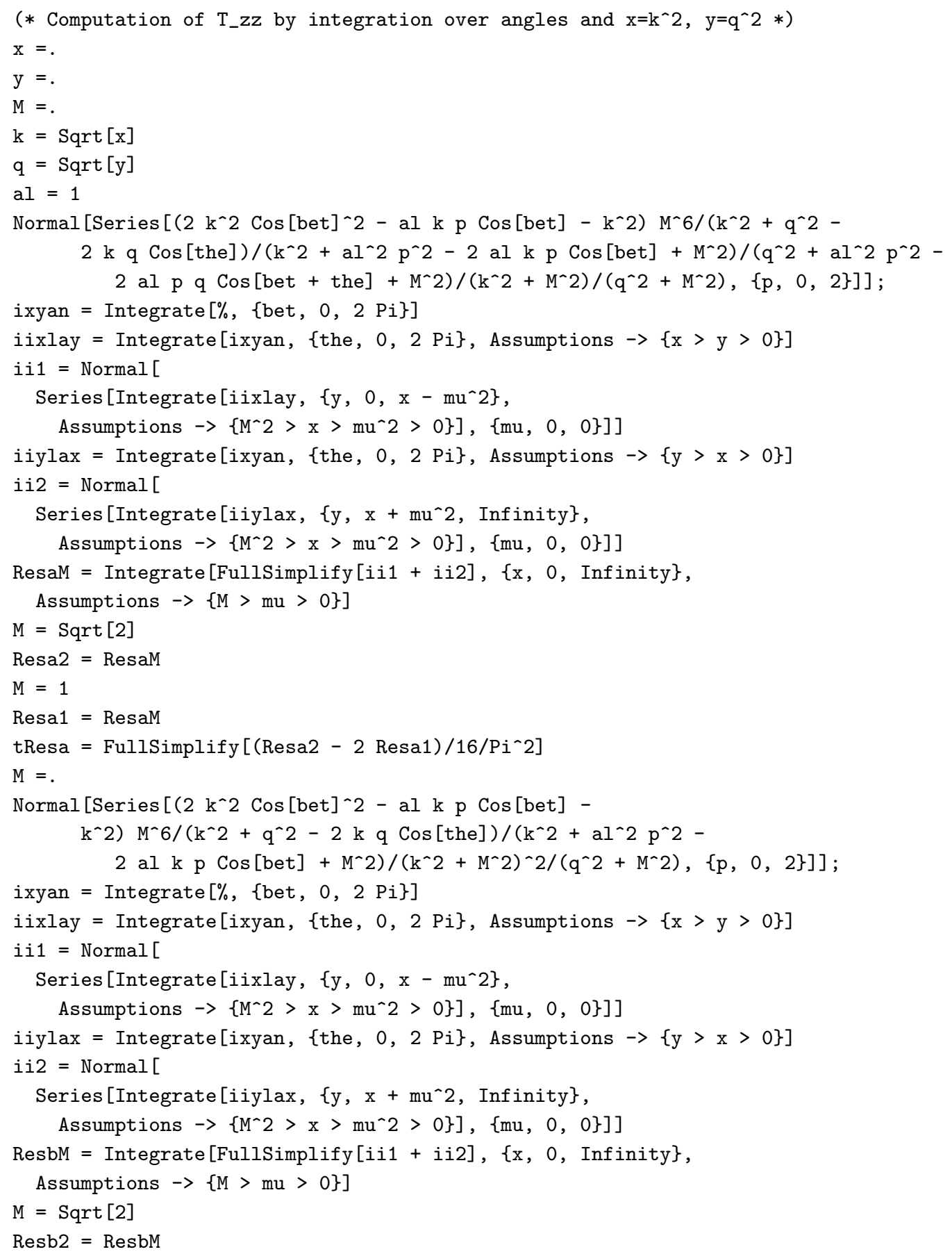




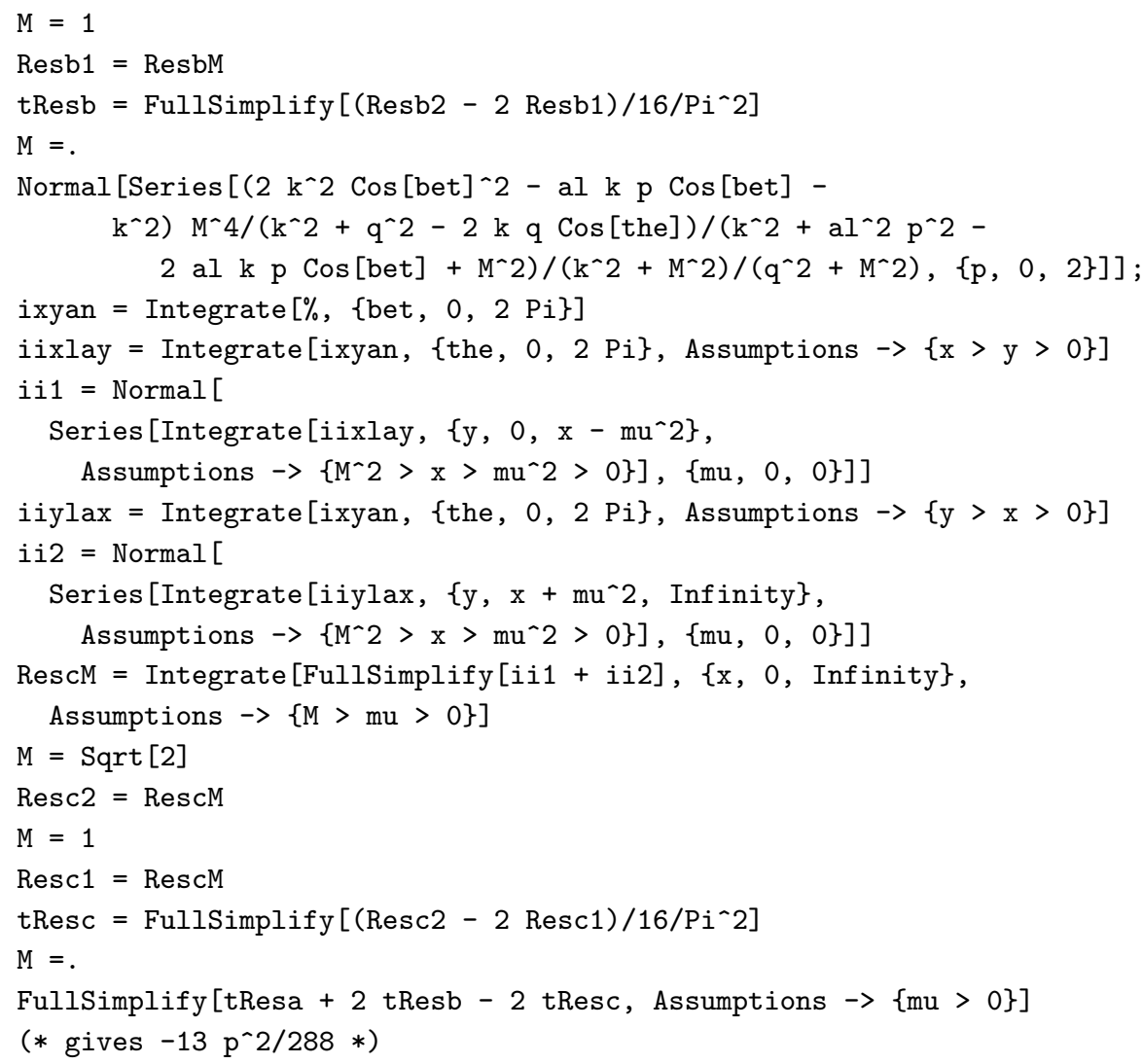

C.2 Program for computing diagrams in figure 4 that results in (8.12)

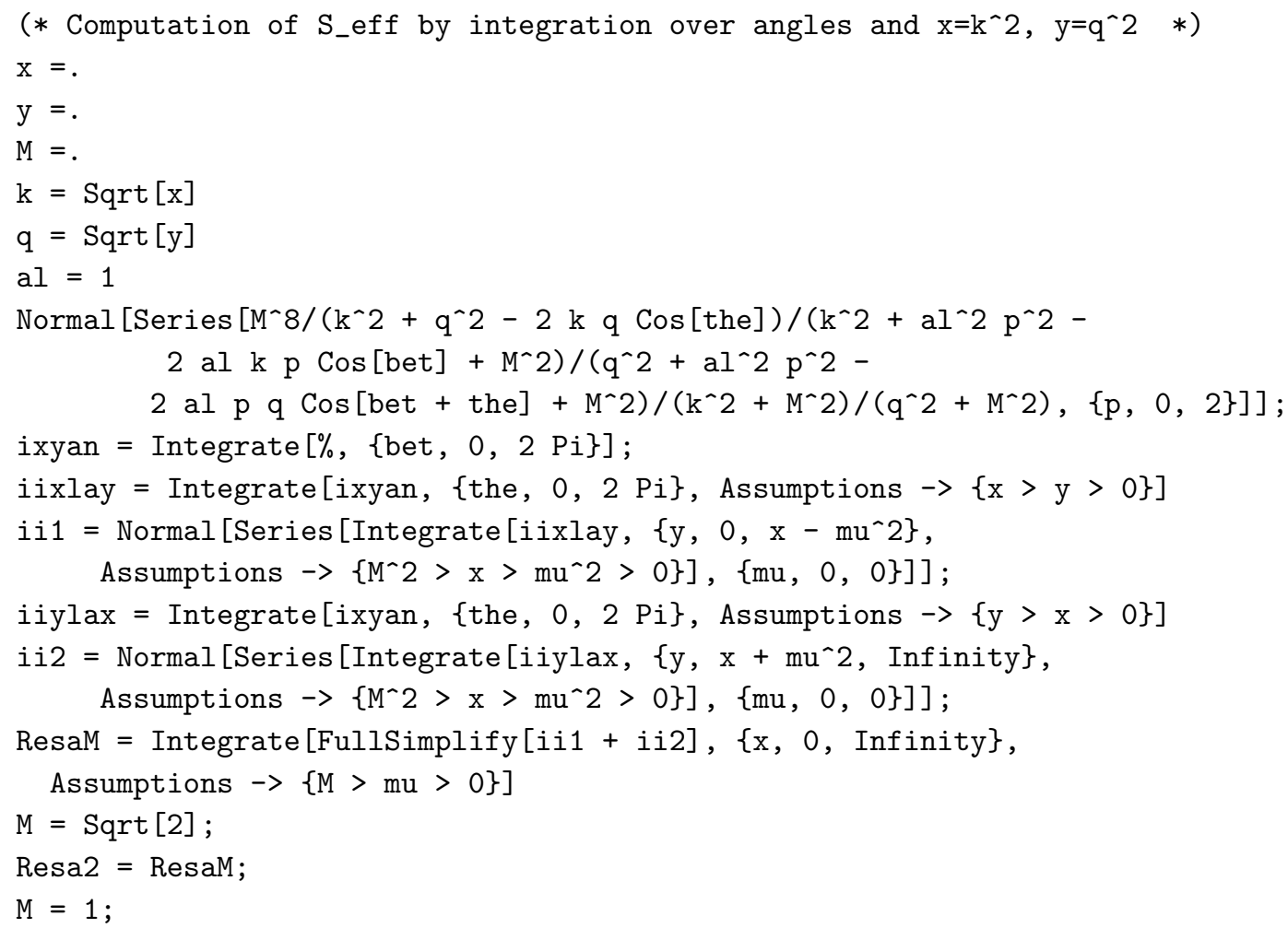




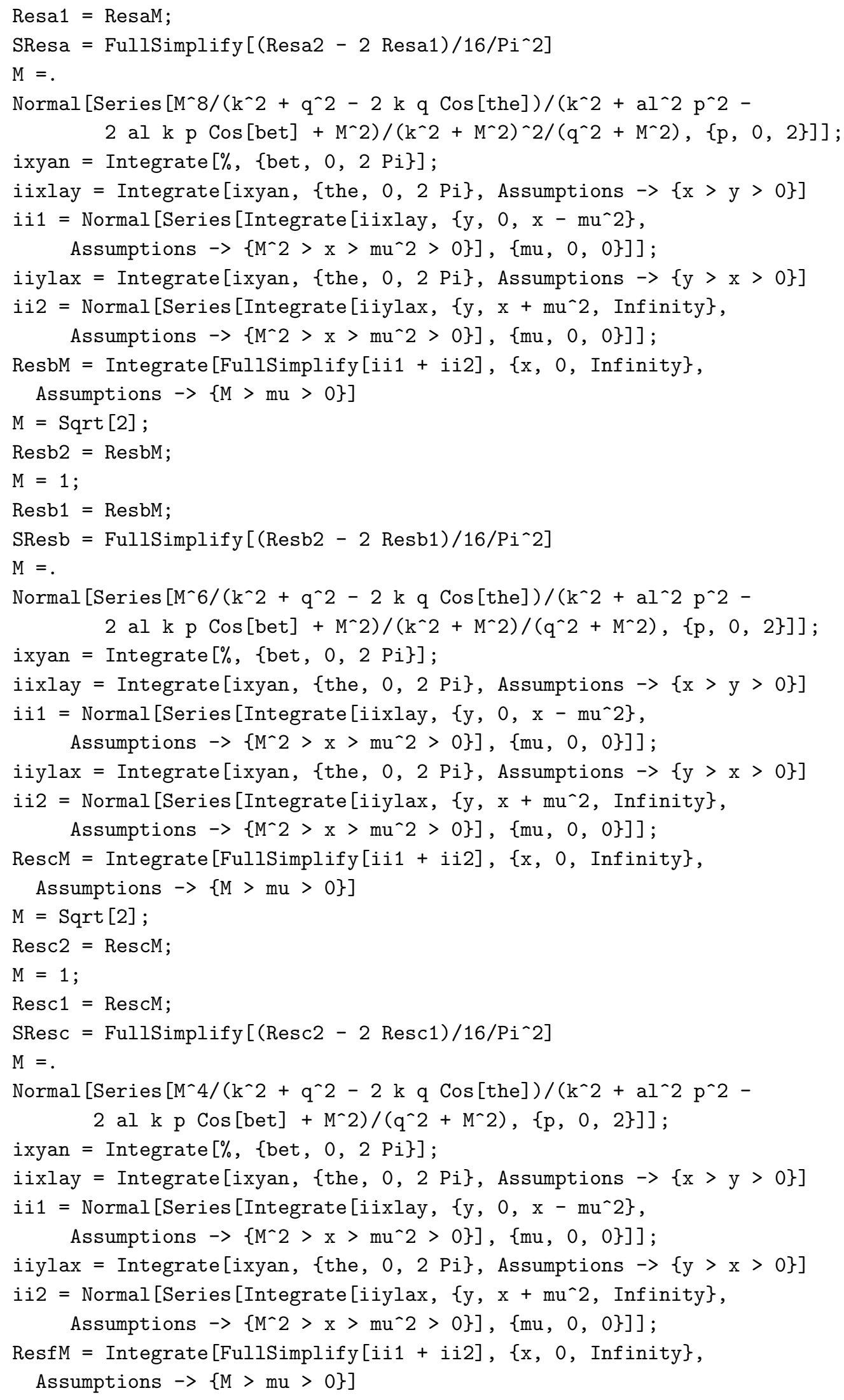




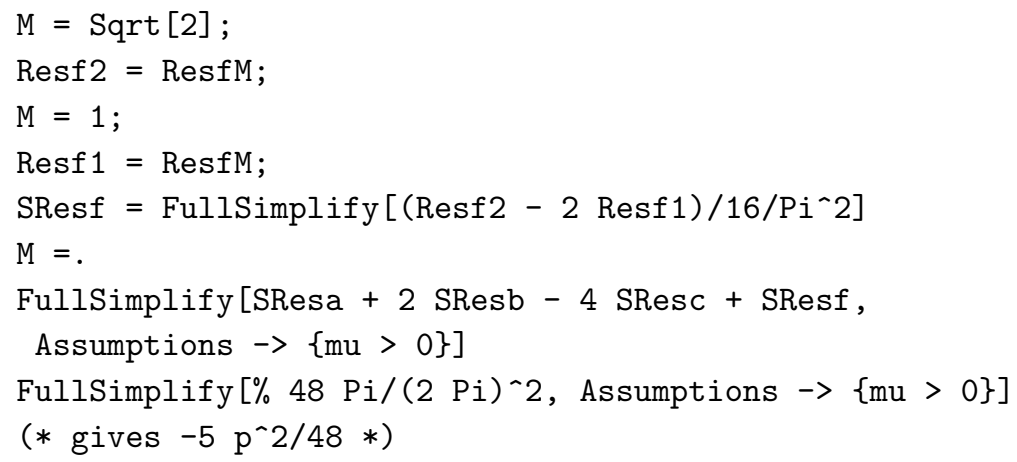

Open Access. This article is distributed under the terms of the Creative Commons Attribution License (CC-BY 4.0), which permits any use, distribution and reproduction in any medium, provided the original author(s) and source are credited.

\section{References}

[1] V.A. Kazakov, A.A. Migdal and I.K. Kostov, Critical Properties of Randomly Triangulated Planar Random Surfaces, Phys. Lett. B 157 (1985) 295 [inSPIRE].

[2] F. David, Planar Diagrams, Two-Dimensional Lattice Gravity and Surface Models, Nucl. Phys. B 257 (1985) 45 [inSPIRE].

[3] J. Ambjørn, B. Durhuus and J. Fröhlich, Diseases of Triangulated Random Surface Models and Possible Cures, Nucl. Phys. B 257 (1985) 433 [InSPIRE].

[4] V.G. Knizhnik, A.M. Polyakov and A.B. Zamolodchikov, Fractal Structure of $2 D$ Quantum Gravity, Mod. Phys. Lett. A 3 (1988) 819 [inSPIRE].

[5] F. David, Conformal Field Theories Coupled to $2 D$ Gravity in the Conformal Gauge, Mod. Phys. Lett. A 3 (1988) 1651 [INSPIRE].

[6] J. Distler and H. Kawai, Conformal Field Theory and 2D Quantum Gravity, Nucl. Phys. B 321 (1989) 509 [INSPIRE].

[7] J. Ambjørn, B. Durhuus and T. Jonsson, Quantum geometry. A statistical field theory approach, Cambridge University Press, Cambridge U.K. (1997).

[8] J. Ambjørn and B. Durhuus, Regularized bosonic strings need extrinsic curvature, Phys. Lett. B 188 (1987) 253 [INSPIRE].

[9] Y. Makeenko, QCD String as an Effective String, in proceedings of the Low dimensional physics and gauge principles, Yerevan, Armenia and Tbilisi, Georgia, 21-29 September 2011, World Scientific (2012), pp. 211-222 [arXiv:1206.0922] [INSPIRE].

[10] J. Polchinski and A. Strominger, Effective string theory, Phys. Rev. Lett. 67 (1991) 1681 [INSPIRE].

[11] S. Dubovsky, R. Flauger and V. Gorbenko, Effective String Theory Revisited, JHEP 09 (2012) 044 [arXiv:1203.1054] [INSPIRE].

[12] O. Aharony and Z. Komargodski, The Effective Theory of Long Strings, JHEP 05 (2013) 118 [arXiv:1302.6257] [INSPIRE].

[13] S. Dubovsky, R. Flauger and V. Gorbenko, Flux Tube Spectra from Approximate Integrability at Low Energies, J. Exp. Theor. Phys. 120 (2015) 399 [arXiv:1404.0037] [INSPIRE]. 
[14] S. Hellerman, S. Maeda, J. Maltz and I. Swanson, Effective String Theory Simplified, JHEP 09 (2014) 183 [arXiv: 1405.6197] [INSPIRE].

[15] B.B. Brandt and M. Meineri, Effective string description of confining flux tubes, Int. J. Mod. Phys. A 31 (2016) 1643001 [arXiv:1603.06969] [INSPIRE].

[16] J.M. Drummond, Universal subleading spectrum of effective string theory, hep-th/0411017 [INSPIRE].

[17] O. Aharony, M. Field and N. Klinghoffer, The effective string spectrum in the orthogonal gauge, JHEP 04 (2012) 048 [arXiv:1111.5757] [INSPIRE].

[18] O. Alvarez, The Static Potential in String Models, Phys. Rev. D 24 (1981) 440 [inSPIRE].

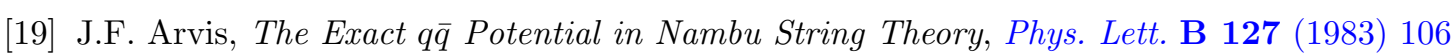
[INSPIRE].

[20] P. Olesen, Strings and QCD, Phys. Lett. B 160 (1985) 144 [INSPIRE].

[21] J. Ambjørn and Y. Makeenko, String theory as a Lilliputian world, Phys. Lett. B 756 (2016) 142 [arXiv: 1601.00540] [INSPIRE].

[22] J. Ambjørn and Y. Makeenko, Scaling behavior of regularized bosonic strings, Phys. Rev. D 93 (2016) 066007 [arXiv: 1510.03390] [INSPIRE].

[23] J. Ambjørn and Y. Makeenko, Stability of the nonperturbative bosonic string vacuum, Phys. Lett. B 770 (2017) 352 [arXiv:1703.05382] [INSPIRE].

[24] J. Ambjørn and Y. Makeenko, The use of Pauli-Villars regularization in string theory, Int. J. Mod. Phys. A 32 (2017) 1750187 [arXiv:1709.00995] [INSPIRE].

[25] A.M. Polyakov, Quantum Geometry of Bosonic Strings, Phys. Lett. B 103 (1981) 207 [INSPIRE].

[26] B. Durhuus, P. Olesen and J.L. Petersen, Polyakov's Quantized String With Boundary Terms, Nucl. Phys. B 198 (1982) 157 [InSPIRE].

[27] O. Alvarez, Theory of Strings with Boundaries: Fluctuations, Topology and Quantum Geometry, Nucl. Phys. B 216 (1983) 125 [InSPIRE].

[28] Y. Makeenko, Methods of contemporary gauge theory, Cambridge University Press, Cambridge U.K. (2002), pp. 208-210.

[29] Y. Makeenko, An interplay between static potential and Reggeon trajectory for QCD string, Phys. Lett. B 699 (2011) 199 [arXiv:1103.2269] [InSPIRE].

[30] L. Brink and H.B. Nielsen, A Simple Physical Interpretation of the Critical Dimension of Space-Time in Dual Models, Phys. Lett. B 45 (1973) 332 [INSPIRE].

[31] A.M. Polyakov, Gauge fields and strings, Harwood Academic Publishers, Reading U.K. (1987), pp. 173-174.

[32] R.C. Brower, Spectrum generating algebra and no ghost theorem for the dual model, Phys. Rev. D 6 (1972) 1655 [inSPIRE].

[33] P. Goddard and C.B. Thorn, Compatibility of the Dual Pomeron with Unitarity and the Absence of Ghosts in the Dual Resonance Model, Phys. Lett. B 40 (1972) 235 [InSPIRE].

[34] A.B. Zamolodchikov, On the entropy of random surfaces, Phys. Lett. B 117 (1982) 87 [INSPIRE]. 
[35] S. Chaudhuri, H. Kawai and S.H.H. Tye, Path Integral Formulation of Closed Strings, Phys. Rev. D 36 (1987) 1148 [inSPIRE].

[36] I.K. Kostov and A. Krzywicki, On the Entropy of Random Surfaces With Arbitrary Genus, Phys. Lett. B 187 (1987) 149 [inSPIRE].

[37] A. Zamolodchikov and A. Zamolodchikov, Lectures on Liouville theory and matrix models, (2008) and online pdf version at http://qft.itp.ac.ru/ZZ.pdf. 